\title{
Nuclear envelope-localized torsinA-LAP1 complex regulates hepatic VLDL secretion and steatosis
}

\author{
Ji-Yeon Shin, ${ }^{1,2}$ Antonio Hernandez-Ono, ${ }^{1}$ Tatyana Fedotova, ${ }^{1}$ Cecilia Östlund, ${ }^{1,2}$ Michael J. Lee, ${ }^{2}$ Sarah B. Gibeley, ${ }^{1}$ Chun-Chi Liang, ${ }^{3}$ \\ William T. Dauer, ${ }^{3,4}$ Henry N. Ginsberg, ${ }^{1}$ and Howard J. Worman ${ }^{1,2}$ \\ 'Department of Medicine, and 2Department of Pathology and Cell Biology, Vagelos College of Physicians and Surgeons, Columbia University, New York, New York, USA. ${ }^{3}$ Department of Neurology, and \\ ${ }^{4}$ Department of Cell and Developmental Biology, University of Michigan Medical School, Ann Arbor, Michigan, USA.
}

\begin{abstract}
Deciphering novel pathways that regulate liver lipid content has profound implications for understanding the pathophysiology of nonalcoholic fatty liver disease (NAFLD) and nonalcoholic steatohepatitis (NASH). Recent evidence suggests that the nuclear envelope is a site of regulation of lipid metabolism, but there is limited appreciation of the responsible mechanisms and molecular components within this organelle. We showed that conditional hepatocyte deletion of the inner nuclear membrane protein lamina-associated polypeptide 1 (LAP1) causes defective VLDL secretion and steatosis, including intranuclear lipid accumulation. LAP1 binds to and activates torsinA, an AAA ${ }^{+}$ATPase that resides in the perinuclear space and continuous main ER. Deletion of torsinA from mouse hepatocytes caused even greater reductions in VLDL secretion and profound steatosis. Mice from both of the mutant lines studied developed hepatic steatosis and subsequent steatohepatitis on a regular chow diet in the absence of whole-body insulin resistance or obesity. Our results establish an essential role for the nuclear envelope-localized torsinA-LAP1 complex in hepatic VLDL secretion and suggest that the torsinA pathway participates in the pathophysiology of NAFLD.
\end{abstract}

\section{Introduction}

Liver triglyceride (TG) metabolism consists of 3 broad processes. First is the accumulation of fatty acids by hepatocytes from the uptake of plasma fatty acids, the uptake of TG fatty acids in remnants of VLDL or chylomicrons, and de novo fatty acid synthesis from glucose. Second is the generation of energy via $\beta$-oxidation of fatty acids within hepatocytes. Third is the storage of TGs in lipid droplets or the delivery of TGs to other tissues in the form of VLDL, an apolipoprotein B-dependent (apoB-dependent) process $(1,2)$. Alterations in 1 or more of these processes may promote hepatic steatosis. Nonalcoholic fatty liver disease (NAFLD) is a burgeoning public health epidemic that is estimated to affect approximately $25 \%$ of the US population (3). In some cases, simple steatosis progresses to nonalcoholic steatohepatitis (NASH), which increases the risk for hepatic fibrosis, cirrhosis, and hepatocellular carcinoma (4).

Many lipid metabolic processes are localized to the ER (5). However, several inherited human diseases, including lipodystrophy syndromes, are caused by mutations in genes encoding proteins localized to the nuclear envelope, a specialized region contiguous with the main ER (6). Mutations in LMNA, encoding lamin $\mathrm{A}$ and lamin $\mathrm{C}$, components of the nuclear lamina

\section{Related Commentary: p. 4576}

Authorship note: WTD, HNG, and HJW are co-senior authors. Conflict of interest: The authors have declared that no conflict of interest exists. Copyright: (5) 2019, American Society for Clinical Investigation. Submitted: April 24, 2019; Accepted: August 7, 2019; Published: October 7, 2019. Reference information: J Clin Invest. 2019;129(11):4885-4900. https://doi.org/10.1172/JCl129769. lining the inner aspect of the inner nuclear membrane, cause Dunnigan-type familial partial lipodystrophy (7-9). Almost all patients with Dunnigan-type familial partial lipodystrophy have hepatic steatosis; however, in this condition it occurs secondarily to adipose dysfunction and insulin resistance (10). Additionally, there are data suggesting that hepatocyte-intrinsic alterations in the nuclear envelope may also directly affect liver lipid metabolism. Conditional deletion of Lmna from hepatocytes causes steatosis and increased susceptibility to steatohepatitis, but for unknown reasons, the effect is only observed in male mice (11). These data implicate the nuclear envelope as a site of regulation of lipid metabolism, but the responsible molecular components and mechanisms within this organelle are poorly understood.

Potential links to lipid metabolism also exist for integral proteins of the inner nuclear membrane. The lamin B receptor is a polytopic inner nuclear membrane protein that is essential for cholesterol synthesis (12). Overexpression in hepatocellular carcinoma of a truncated variant of lamina-associated polypeptide $2 \alpha$, a nucleocytoplasmic isoform of an inner nuclear membrane protein, alters fatty acid uptake (13). Deletion of the laminaassociated polypeptide 1 (LAP1), a monotopic protein of the inner nuclear membrane (actually 3 different isoforms with variable nucleoplasmic domains encoded by the same gene), causes an apparent expansion of the inner nuclear membrane, suggestive of altered lipid metabolism (14).

The nucleoplasmic domain of LAP1 interacts with nuclear lamins and emerin, another integral protein of the inner nuclear membrane $(15,16)$. The luminal domain of LAP1, however, binds to torsin $\mathrm{A}$, an $\mathrm{AAA}^{+}$ATPase that resides in the perinuclear space and the continuous main ER (17). Consistent with these data, torsinA is enzymatically inactive unless it interacts with LAP1 
within the perinuclear space or with the luminal domain of the transmembrane protein lumenal domain-like LAP1 (LULL1) within the main ER $(17,18)$. Recessive mutations in the gene encoding LAP1 that disrupt the B isoform have been linked to familial cardiomyopathy and muscular dystrophy $(19,20)$. Total loss of LAP1B and LAP1C causes multisystem disease and death during childhood (21). A single amino acid deletion in torsinA causes the neurodevelopmental disease DYT1 dystonia, which is an autosomal dominant disease (22). Despite these multiple disease associations, the role of the torsinA-LAP1 complex in different tissues remains unestablished.

To unravel fundamental features of the torsinA-LAP1 complex, we have explored the molecular and cellular consequences of disrupting these proteins in diverse tissue environments (16, 23). Here, we report that conditional deletion of either LAP1 or torsinA from hepatocytes caused profound steatosis and establish a critical role for the torsinA-LAP1 complex in hepatic VLDL assembly and secretion. These data advance our understanding of nuclear envelope-localized processes in lipid homeostasis and are the first to our knowledge to implicate the torsinA-LAP complex in mammalian lipid metabolism.

\section{Results}

Conditional hepatocyte deletion of LAP1 causes hepatic steatosis. We reported previously that chow-fed mice in which LAP1 was conditionally deleted from hepatocytes $\left(\mathrm{Alb}-\mathrm{Cre}^{+/-} \mathrm{Lap}{ }^{1 \mathrm{fl} / \mathrm{l}}\right.$, hereafter referred to L-CKO mice) were grossly indistinguishable from littermate controls up to almost 2 years of age (16). However, although the livers of L-CKO mice were grossly normal at 4 months of age, their hepatocytes had enlarged nuclei (16). Using electron microscopy to further investigate the enlarged nuclei in L-CKO mice, we observed large ovoid structures within hepatocyte nuclei that appeared to be lipid droplets (in chow-fed male L-CKO mice; Figure 1A). Confocal fluorescence micrographs of isolated hepatocytes from L-CKO mice stained with the lipophilic fluorescent dye boron-dipyrromethene (BODIPY) confirmed the presence of large intranuclear lipid droplets in L-CKO nuclei and increased numbers of cytosolic lipid droplets that appeared somewhat larger, on average, than those in control hepatocytes (Figure 1B). In contrast, hepatocytes from $E m d^{-/ y}$ mice lacking emerin, an integral protein of the inner nuclear membrane that interacts with LAP1, did not exhibit intranuclear lipid droplets (data not shown). $\mathrm{H} \& \mathrm{E}$-stained sections of livers from 6-month-old male L-CKO mice fed a regular chow diet demonstrated cytoplasmic fat vacuoles, which was confirmed by staining with Oil Red O (Figure 1C). L-CKO livers contained significantly increased liver TG and cholesterol levels compared with livers of their littermate controls (Figure 1D). Steady-state plasma TG concentrations did not differ significantly from those of controls, but the plasma cholesterol concentration was significantly decreased in L-CKO mice at 6 months of age (Figure 1E). L-CKO mice exhibited normal glucose tolerance (Figure $1 \mathrm{~F}$ ) and fasting serum insulin concentrations (Figure 1G), indicating normal whole-body insulin sensitivity. Similarly aged female L-CKO mice had steatosis and a metabolic profile that was indistinguishable from that of their male counterparts (Supplemental Figure 1; supplemental material available online with this article; https://doi.org/10.1172/JCI129769DS1).
We reported previously that the body mass of L-CKO mice did not differ from that of controls up to approximately 2 years of age (16). Further body composition analysis of 4- to 6-month-old male and female L-CKO mice fed a chow diet did not show any differences in the percentage of body fat compared with controls (Supplemental Figure 2A). The percentage of lean body mass was also the same in L-CKO and control mice of both sexes (Supplemental Figure 2B). Collectively, our results indicate that depletion of LAP1 from hepatocytes of male and female mice causes hepatic steatosis and nuclear lipid droplets. Steatosis occurred in mice on a chow diet in the absence of obesity, glucose intolerance, wholebody insulin resistance, or changes in body composition. Because none of the identified phenotypes showed sexual dimorphism, in all subsequent experiments we analyzed L-CKO data from both sexes in aggregate.

Defective hepatic TG and apoB10O secretion in L-CKO mice. To gain a mechanistic understanding of hepatic steatosis in L-CKO mice, we investigated de novo hepatic lipogenesis, fatty acid oxidation, and lipoprotein secretion. Analysis of the expression of selected genes encoding proteins involved in liver lipid metabolism failed to identify a specific pathway to explain steatosis in the L-CKO mice. Examination of the expression of key genes in de novo lipogenesis provided a mixed picture: Fasn, encoding fatty acid synthase, and Dgat2, encoding diacylglycerol $O$-acyltransferase 2, were significantly reduced, whereas expression of Srebf1, Srefb2, Acaca, Scd1, and Dgat1 did not differ significantly between L-CKO and littermate controls (Supplemental Figure 3). We therefore measured de novo hepatic lipogenesis in whole livers and fatty acid oxidation in isolated primary hepatocytes from L-CKO and control mice. We observed no significant abnormalities in either hepatic de novo lipogenesis (Figure $2 \mathrm{~A}$ ) or fatty acid oxidation in L-CKO mice (Figure 2B). It is unlikely that the supply of fatty acids from adipose tissue would have been changed in the L-CKO mice, as they have hepatocyte-selective gene deletion. Nonetheless, we examined plasma fatty acid concentrations to exclude increased peripheral delivery to the liver and found that the concentrations were the same in L-CKO and control mice (Figure 2C). These results indicate that increased synthesis, decreased oxidation, or increased delivery of circulating fatty acids was not contributing to hepatic steatosis in L-CKO mice.

Another potential mechanism for steatosis is a defect in hepatic lipoprotein secretion. Consistent with this possibility, we found that plasma TG concentrations were reduced in 4-month old chow-fed L-CKO mice at all time points measured following i.v. administration of tyloxapol to block uptake of circulating TG-rich lipoproteins (Figure 3A). The hepatic TG secretion rate calculated from those data was significantly reduced by $20.1 \% \pm$ $7.4 \%$ in L-CKO mice (Figure 3B). apoB is essential for the assembly and secretion of VLDL. Although human livers synthesize and secrete only apoB100, rodents also synthesize and secrete an alternatively translated, truncated form, apoB48, which is also made in human and rodent small intestines. The role of hepatic apoB48 is unclear, but it can be the dominant form in rodent livers (24). Analysis of plasma proteins by SDS-PAGE followed by gel autoradiography 120 minutes after injection of ${ }^{35} \mathrm{~S}$-methionine together with tyloxapol showed decreased levels of radiolabeled apoB100 in L-CKO mice, but similar amounts of 
A

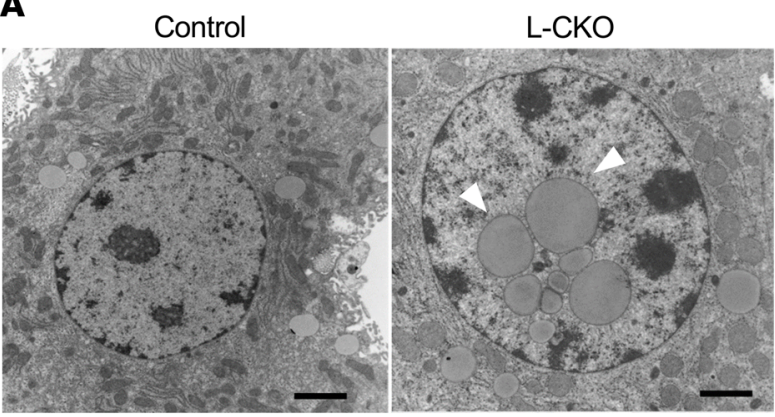

B
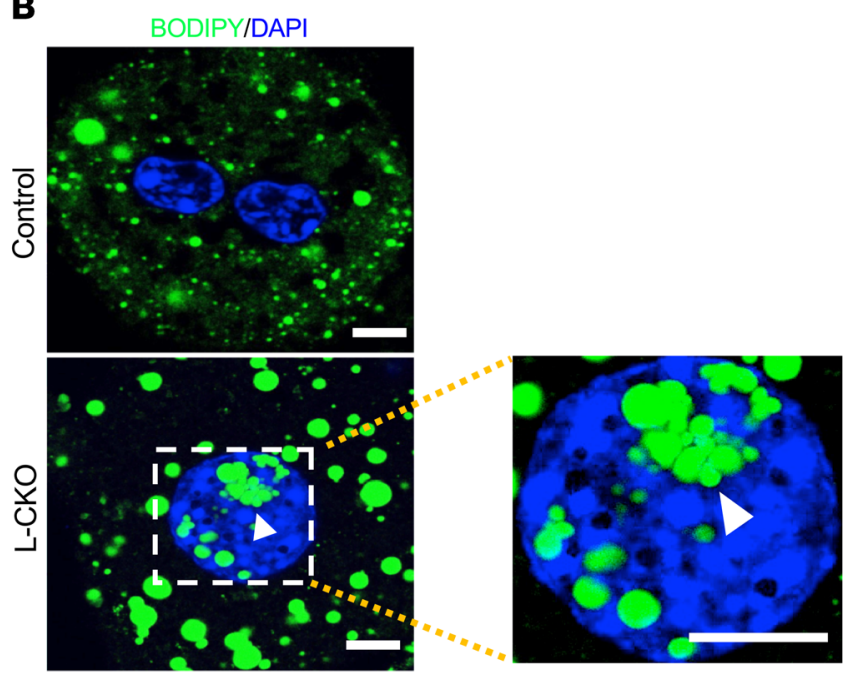

C
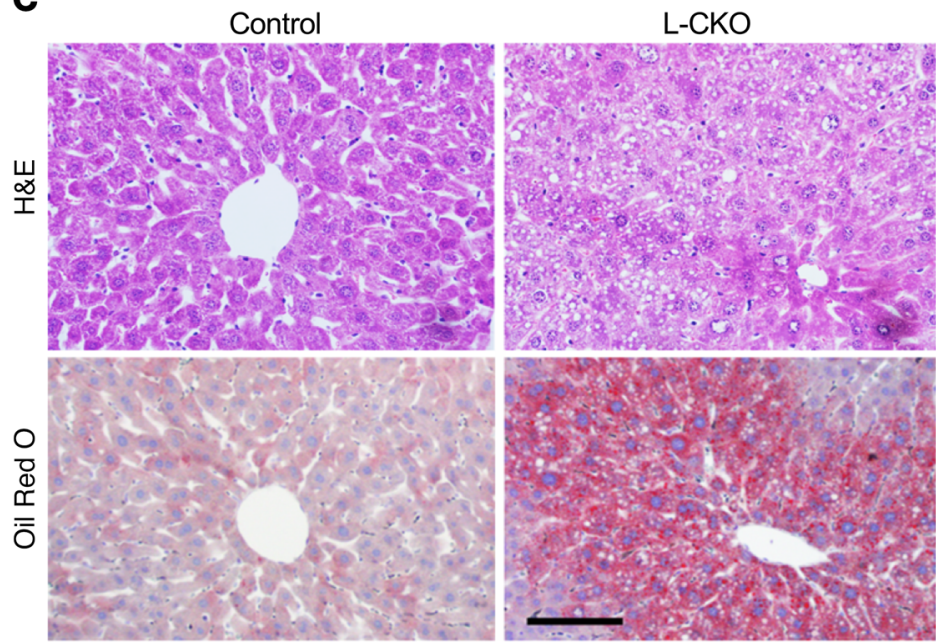
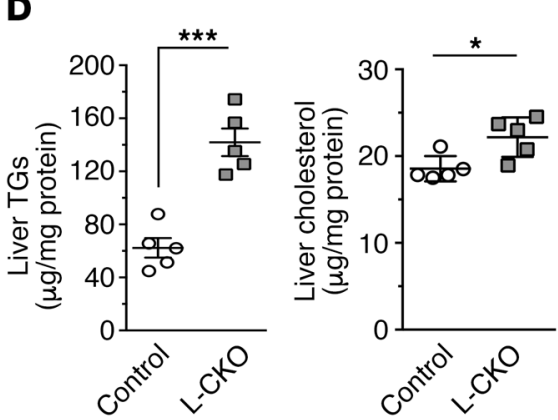

$\mathbf{E}$

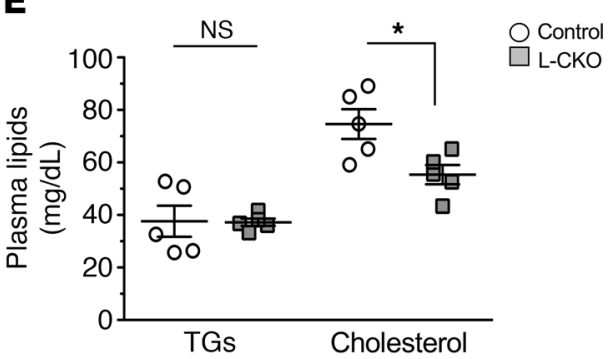

$\mathbf{F}$

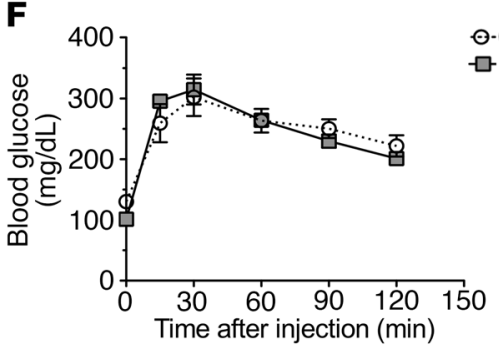

G

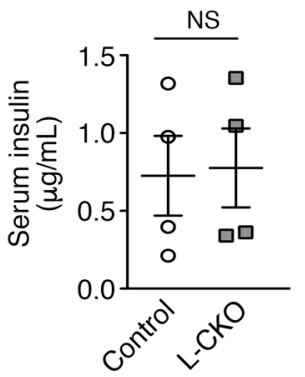

Figure 1. Hepatic steatosis without evidence of insulin resistance in male L-CKO mice fed a chow diet. (A) Electron micrographs of liver sections from male control (Tor1aip $\left.f^{f / f}\right)$ and L-CKO (AlbCre Tor1aip $\left.f^{f / f}\right)$ mice. White arrowheads indicate intranuclear lipid droplets. Scale bars: $2 \mu \mathrm{m}$. (B) Confocal micrographs of isolated hepatocytes. Lipids were stained with BODIPY (green) and nuclei with DAPI (blue). The right panel is a zoomed image of the dashed-line square region. White arrowheads indicate intranuclear lipid droplets. Scale bars: $10 \mu \mathrm{m}$ (zoom, $10 \mu \mathrm{m})$. (C) Representative light micrographs of H\&E- and Oil Red 0-stained liver sections from chow-fed mice. Scale bar: $50 \mu \mathrm{m}$. (D) Liver TC and cholesterol content; mice were fasted for 4 to 5 hours before livers were collected ( $n=5$ mice per group). ${ }^{*} P<0.05$ and ${ }^{* * *} P<0.001$, by Student's $t$ test. (E) Plasma TC and cholesterol concentrations. Mice were fasted for 5 hours before collection of plasma ( $n=5$ mice per group). ${ }^{*} P<0.05$, by Student's $t$ test. (F) Blood glucose concentration versus time after injection of a glucose bolus in overnight-fasted mice. Values represent the mean \pm SEM ( $n=6$ mice per group). Results were not significantly different at any time point by ANOVA. (G) Serum insulin concentrations. Mice were fasted for 5 hours before collection of plasma ( $n=4$ mice per group). $P=N S$, by Student's $t$ test. In D, E, and $\mathbf{G}$, the values for individual mice are shown, with longer horizontal bars indicating the mean and vertical bars indicating the SEM. The control and L-CKO mice used were 4-6 months of age. 

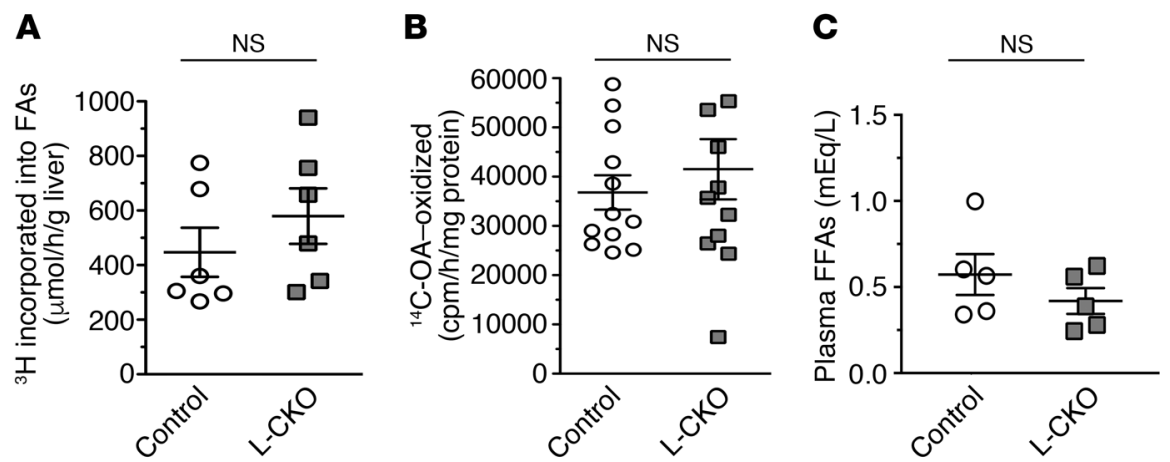

Figure 2. De novo lipogenesis, fatty acid oxidation, and plasma free fatty acids in livers of L-CKO mice. (A) De novo lipogenesis in the livers of control and L-CKO mice. Newly synthesized hepatic lipids were measured by incorporation of ${ }^{3} \mathrm{H}$ into fatty acids (FAs) 1 hour after i.p. injection with ${ }^{3} \mathrm{H}_{2} \mathrm{O}(n=6$ mice per genotype). $P=$ NS, by Student's $t$ test. (B) Oxidation of fatty acids in hepatocytes isolated from control and L-CKO mice. Oxidative products of ${ }^{14} \mathrm{C}$-oleic acid $\left({ }^{14} \mathrm{CO}_{2}\right.$ and ${ }^{14} \mathrm{C}$-labeled acid-soluble metabolites) were measured and normalized to milligrams of cellular protein ( $n=3$ mice per genotype). $P=$ NS, by Student's $t$ test. (C) Free fatty acids (FFAs) were measured from snap-frozen plasma samples from control and L-CKO mice after a 4 - to 5 -hour fast. The values for individual mice are shown, with longer horizontal bars indicating the mean and vertical bars indicating the SEM. Mice used were 4-6 months of age.

the alternatively translated product apoB48 (Figure 3C). Secretion of newly synthesized apoB100 was reduced by $33.1 \% \pm 5.4 \%$ in L-CKO mice compared with controls, and apoB48 secretion was normal in L-CKO mice (Figure 3D). To exclude extrahepatic influences on apoB100 secretion in L-CKO mice, we incubated primary cultures of hepatocytes isolated from L-CKO and control mice with ${ }^{35} \mathrm{~S}$-methionine and performed autoradiography of SDS-polyacrylamide gels in which proteins in cell lysates and media were separated (Figure $3 \mathrm{E}$ ). As with the in vivo findings, we observed a significant and selective $23.4 \% \pm 6.4 \%$ decrease in apoB100 in media harvested from these cells. Analysis of the whole-cell lysates showed a small but significant decrease in apo48 as well as a larger, significant decrease in apoB100 (Figure $3 \mathrm{~F})$. These data demonstrate that $\mathrm{L}-\mathrm{CKO}$ mice have a selective defect in the secretion of apoB100 VLDL.

To assess whether the defect in TG and apoB100 secretion was specific, or reflected a general abnormality of hepatic secretory function, we measured the secretion of newly synthesized serum albumin and apoA1 by autoradiography in the same in vivo studies in which we measured apoB secretion (Supplemental Figure $4 \mathrm{~A})$. We observed no significant differences for secretion of either protein in L-CKO mice (Supplemental Figure 4B). The steadystate serum albumin concentration was also normal in L-CKO mice (Supplemental Figure 4C). These data exclude a general secretory defect in L-CKO hepatocytes and point to a specific defect of apoB100 secretion caused by the absence of LAP1.

The Alb-Cre transgene used to delete LAP1 in L-CKO mice closely mimics the expression of endogenous $A l b$, which begins on E10.5 (25). To exclude the possibility that the phenotypes of these mice result from developmental effects, we deleted LAP1 from hepatocytes of adult mice by injecting a Cre-expressing adenoassociated virus (AAV) construct (AAV-Cre) into the tail veins of adult mice homozygous for floxed alleles of the gene encoding LAP1. LAP1 levels were reduced by approximately $50 \%$ three weeks after virus injection and were largely undetectable 8 weeks after injection (Figure 4A). As seen with hepatocytes from L-CKO mice, primary hepatocytes isolated from mice injected with AAV-Cre exhibited increased numbers of larger cytoplasmic lipid droplets, compared with control hepatocytes, as well as intranuclear lipid droplets (Figure $4 \mathrm{~B})$. Synthesis and secretion of apoB in primary cultures of hepatocytes from AAVCre-injected mice after incubation with ${ }^{35} \mathrm{~S}$-methionine also mimicked the findings in L-CKO mice (Figure 4C). We observed a significant decrease in apoB100 in cell lysates and media $(72.8 \% \pm 9.2 \%$ and $56.2 \% \pm 12.9 \%$, respectively) of AAV-Creinfected primary hepatocytes, whereas apoB48 was slightly but significantly decreased $(23.4 \% \pm 6.4 \%)$ in cell lysates but unchanged in the media (Figure 4D). These data confirm an essential role for LAP1 in hepatocyte lipid metabolism and exclude the possibility that the phenotype of L-CKO mice results from an early developmental defect.

Conditional hepatocyte deletion of torsinA causes hepatic steatosis. The luminal domain of LAP1 binds to the $\mathrm{AAA}^{+}$ATPase torsinA within the perinuclear space of the nuclear envelope (17). LAP1 binding is required for torsinA ATPase activity (18). We first excluded the possibility that loss of LAP1 decreases torsinA levels or dramatically alters its localization in hepatocytes. When we examined the livers of L-CKO mice for torsinA expression, there was actually a slight but significant increase in torsin A expression compared with that in the livers of control mice (Supplemental Figure 5, A and B). TorsinA continued to have an ER-like distribution in hepatocytes lacking LAP1, as in control hepatocytes (Supplemental Figure 5C). We therefore hypothesized that loss of LAP1 induces hepatic steatosis by decreasing torsinA activity at the nuclear envelope. We tested this hypothesis through conditional deletion of torsinA from hepatocytes by intercrossing Tor1 $a^{f l-}$ (23) and Alb-Cre-transgenic mice (referred to herein as A-CKO mice), predicting that these mice would also have hepatic steatosis and decreased VLDL section.

A-CKO mice were born at the expected Mendelian ratios and were indistinguishable from their littermate controls at birth. We confirmed the efficiency of torsinA depletion by immunoblotting of proteins in lysates of liver and primary hepatocytes from A-CKO mice (Supplemental Figure 6, A and B). Adult male A-CKO mice fed a chow diet exhibited grossly enlarged, whitish livers (Figure 5A). H\&E-stained sections of livers from A-CKO mice had grossly enlarged hepatocytes with mixed macrovesicular and microvesicular steatosis, which was confirmed with Oil Red O staining (Figure 5B). Livers from A-CKO mice contained dramatically increased amounts of TGs and cholesterol compared with littermate controls; levels of both lipids were much higher than those in the livers of L-CKO mice (Figure 5C). Concomitant with the much greater steatosis, A-CKO mice had markedly reduced plasma TG and cholesterol concentrations (Figure 5D). Despite the more dramatic hepatic steatosis and reductions in plasma lipid levels 
A

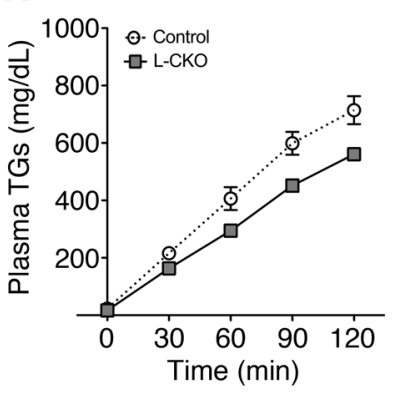

B

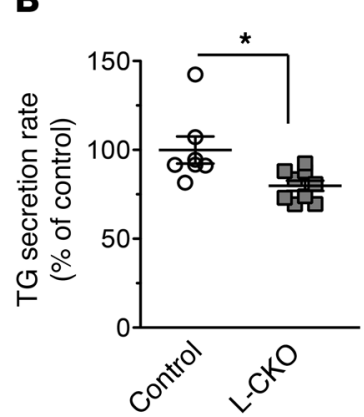

C

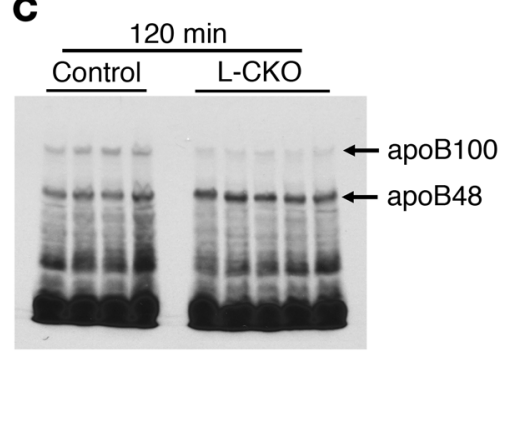

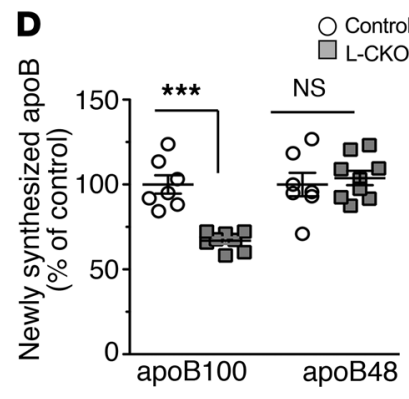

E

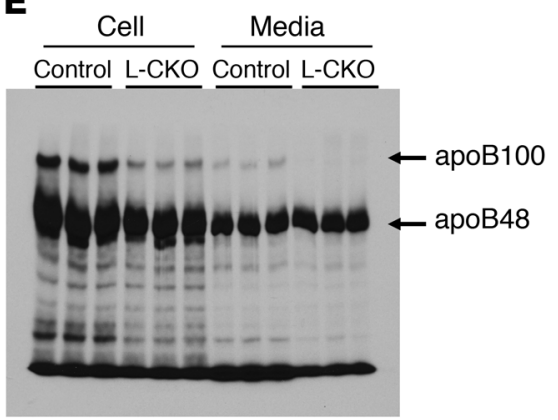

$\mathbf{F}$

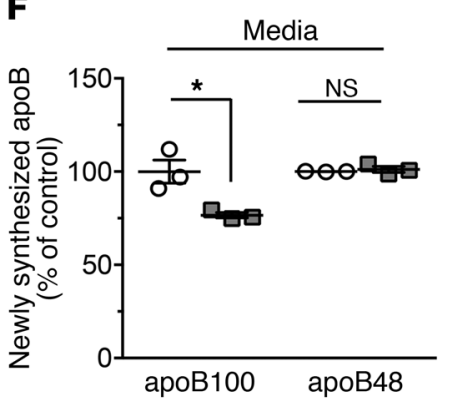

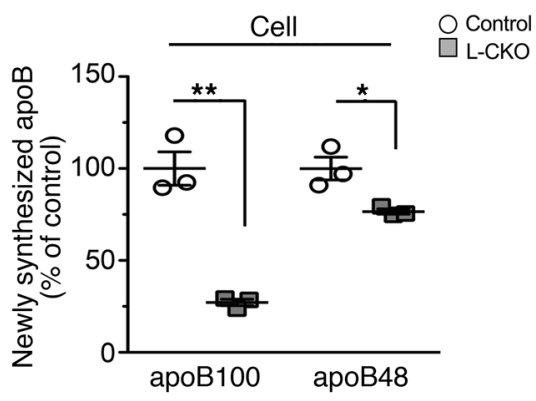

Figure 3. Reduced TG and apoB100 secretion from livers and isolated hepatocytes of L-CKO mice. (A) Mice were injected with tyloxapol to block peripheral TC uptake, and plasma concentrations were measured at the indicated time points. Values indicate the mean \pm SEM ( $n=6-9$ mice per group). (B) TC secretion rates calculated from the changes in plasma concentrations between 30 and 120 minutes in $\mathbf{A} .{ }^{*} P<0.05$, by Student's $t$ test. (C) Autoradiogram of SDS-polyacrylamide gel showing ${ }^{35} \mathrm{~S}$-labeled plasma proteins collected 120 minutes after injection with ${ }^{35} \mathrm{~S}$-methionine. Each lane shows proteins from an individual mouse. Migrations of ${ }^{35} \mathrm{~S}$-methionine-labeled apoB100 and apoB48 are indicated. (D) Bands corresponding to apoB100 and apoB48 were cut from the gel shown in $\mathbf{C}$, and radioactivity was measured by scintillation counting. Two sets of experiments were combined for the final results ( $n=7-9$ mice per group). ${ }^{* *} P<0.001$, by Student's $t$ test. (E) Autoradiogram of SDS-polyacrylamide gel showing newly synthesized ${ }^{35} S$-labeled proteins in cell lysates and media fractions of primary hepatocyte cultures. Migrations of ${ }^{35} \mathrm{~S}$-labeled apoB100 and apoB48 are indicated ( $n=3$ different hepatocyte cultures from 1 mouse of each genotype). (F) Bands corresponding to apoB100 and apoB48 were cut from the gel shown in E, and radioactivity was quantified by scintillation counting. The left panel shows results from the media fraction (Media) and the right panel from the cell lysate fraction (Cell). Results were normalized to the mean values from control samples, which were set to $100 \%$ ( $n=3$ different hepatocyte cultures from 1 mouse of each genotype). ${ }^{*} P<$ 0.05 and ${ }^{* *} P<0.01$, by Student's $t$ test. In panels $\mathbf{B}, \mathbf{D}$, and $\mathbf{F}$, the values for individual mice are shown, with longer horizontal bars indicating the mean and vertical bars indicating the SEM. Mice used were 4 months of age.

in A-CKO mice compared with L-CKO mice, similar to L-CKO mice, the A-CKO mice had normal glucose tolerance (Figure 5E) and fasting serum insulin concentrations (Figure 5F). Electron microscopy revealed numerous electron-dense, vesicle-like structures throughout the cytoplasm of A-CKO hepatocytes (Figure $5 \mathrm{G})$. Confocal fluorescence micrographs of isolated hepatocytes stained with BODIPY showed the accumulation of numerous small lipid droplets that appeared to be arranged in tubular configurations, suggesting that they may be in the ER (Figure 5H). We did not observe intranuclear lipid droplets in hepatocytes from A-CKO mice. Female A-CKO mice exhibited all of the aforementioned phenotypes of the male animals (Supplemental Figure 7).

The body mass of male or female A-CKO mice did not differ from that of littermate controls for at least the first 6 months of life (Supplemental Figure 8, A and B). Likewise, we observed no significant differences between A-CKO mice and littermate controls in the percentages of body fat or lean body mass (Supplemental Figure 8, C and D). Because the hepatic, metabolic, growth, and body mass phenotypes did not show sexual dimorphism, we analyzed data from animals of both sexes in an aggregate manner in all subsequent experiments.
Defective hepatic TG and apoB10O secretion in A-CKO mice. To further assess the hypothesis that the phenotype of L-CKO mice reflects deficient torsinA function, we examined whether A-CKO mice exhibit similar defects in apoB metabolism. These analyses demonstrated that, concomitant with the greater steatosis and more marked reductions in plasma lipid levels in the A-CKO mice, they had greater reductions in plasma TG levels at all time points measured following i.v. administration of tyloxapol to block the uptake of circulating TG-rich lipoproteins (Figure 6A). The calculated hepatic TG secretion rate for A-CKO mice was markedly and significantly reduced (by $63.8 \% \pm 12.3 \%$; Figure $6 \mathrm{~B}$ ). We analyzed plasma protein levels after injection of ${ }^{35} \mathrm{~S}$-methionine into A-CKO mice (Figure 6C). Compared with control mice, A-CKO mice exhibited significantly decreased apoB100 secretion compared with controls (reduced by $61.3 \% \pm 11.3 \%$ ). As with L-CKO mice, secretion of apoB48 in A-CKO mice did not differ from that detected in controls (Figure 6D). The reduction in apoB100 secretion in A-CKO mice was clearly greater than that observed in L-CKO mice. We also analyzed the synthesis and secretion of apoB in primary hepatocyte cultures from A-CKO mice after incubation with ${ }^{35} \mathrm{~S}$-methonine (Figure $6 \mathrm{E}$ ). This analysis showed sig- 
A

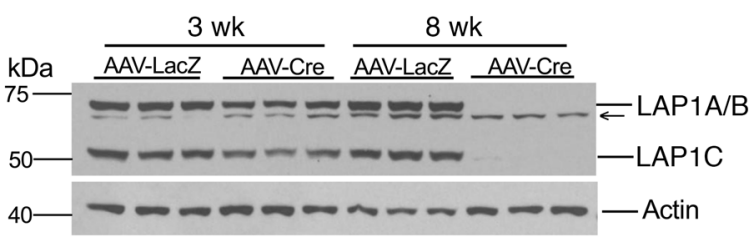

B

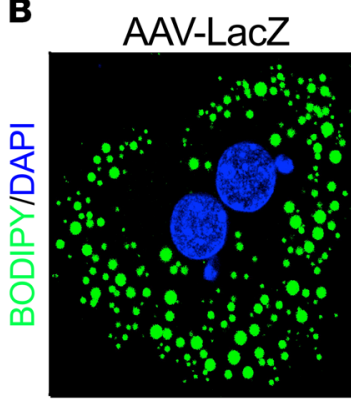

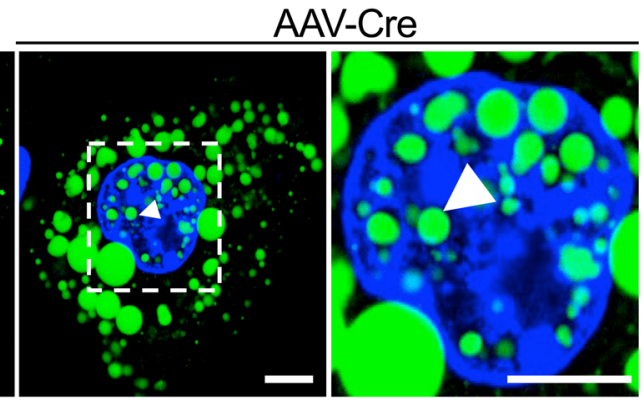

C

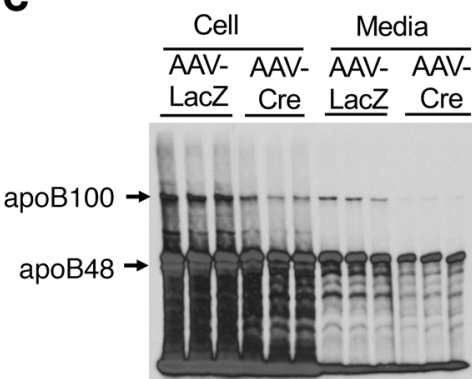

D

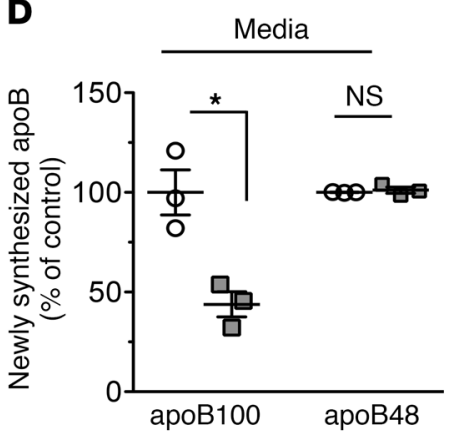

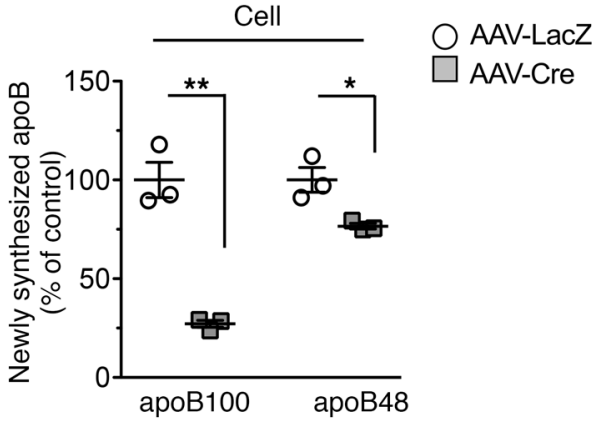

Figure 4. Intranuclear and increased cytoplasmic lipid droplets and reduced apoB100 secretion in hepatocytes isolated from adult mice with "acute" depletion of LAP1. (A) Immunoblot of hepatocyte protein lysates from Tor1aip $7^{f / f f}$ mice injected with AAV-LacZ (control) or AAV-Cre probed with antibodies against LAP1. Hepatocytes were isolated 3 weeks and 8 weeks after virus injection. Each lane represents a primary culture of hepatocytes from 1 mouse. Migrations of molecular mass standards are indicated at the left and migrations of LAP1A/LAP1B and LAP1C at the right. The arrow points to the nonspecific band. An immunoblot for actin is shown as a loading control. (B) Confocal micrographs of isolated hepatocytes from Tor1aip $7^{f l / f l}$ mice injected with AAV-LacZ or AAV-Cre. Lipids were stained with BODIPY (green) and nuclei with DAPI (blue). The far-right panel is a zoomed image of the dashedline square region. White arrowheads indicate intranuclear lipid droplets. Scale bar: $10 \mu \mathrm{m}(\mathrm{zoom}, 10 \mu \mathrm{m})$. (C) Autoradiogram of SDS-polyacrylamide gel showing newly synthesized ${ }^{35} \mathrm{~S}$-methionine-labeled proteins in cell lysates and media fractions of primary hepatocytes cultures from Tor1aip $7^{f / f l}$ mice injected with AAV-LacZ or AAV-Cre. Migrations of ${ }^{35}$ S-labeled apoB100 and apoB48 are indicated. (D) Bands corresponding to apoB100 and apoB48 were cut from the gel shown in $\mathbf{C}$, and radioactivity was quantified by scintillation counting. The left panel shows results from the media fraction and the right panel from the cell fraction. Results were normalized to the mean values from AAV-LacZ-injected mice and set to $100 \%$ ( $n=3$ different hepatocyte cultures from 1 mouse of each group. ${ }^{*} P<0.05$ and ${ }^{* *} P<0.01$, by Student's $t$ test. In $\mathbf{D}$, the values for individual mice are shown, with longer horizontal bars indicating the mean and vertical bars indicating the SEM. Mice were 4 months old at the time of virus injection.

nificantly decreased levels of newly synthesized apoB100 (86.7\% $\pm 11.8 \%)$ and apoB48 $(30.6 \% \pm 6.5 \%)$ in media (Figure $6 \mathrm{~F})$. In cell lysates, we detected a significant decrease in apoB100 (by $62.0 \%$ $\pm 9.3 \%$ ), but no significant changes in newly synthesized apoB48 (Figure 6F). These results indicate that the secretion of both apoB100 and apoB48 is decreased in hepatocytes of A-CKO mice.

Like L-CKO mice, A-CKO mice exhibited no abnormalities in the rate of hepatic secretion of albumin or apoA1 (Supplemental Figure 9, A and B). Serum albumin concentrations were also normal in A-CKO mice (Supplemental Figure 9C). These data indicate that, as in L-CKO mice, there was no general synthetic or secretory defect in the hepatocytes of A-CKO mice.

Because of the profound steatosis and decreased VLDL section in A-CKO mice, we examined livers from mice heterozygous for Tor1a deletion, with 1 allelic expression of torsinA in hepatocytes (A-CKO het). Control and A-CKO het mice did not show significant hepatocyte lipid accumulation, in contrast to A-CKO mice (Supplemental Figure 10A). Liver TG and cholesterol content and plasma concentrations were also similar to those of WT controls (Supplemental Figure 10, B and C). Secretion of hepatic TG and apoB was also identical to that seen in control mice (Supplemental Figure 10, D and E). Hence, heterozygous loss of torsin A from hepatocytes did not lead to steatosis or significantly decreased liver VLDL secretion.

As with L-CKO mice, we examined the expression of selected genes encoding proteins involved in liver lipid metabolism as well as de novo lipogenesis and fatty acid oxidation in the livers of A-CKO mice. Whereas L-CKO mice had reduced hepatic expression of Fasn and Dgat2, both of which are involved in de novo lipogenesis, A-CKO mice showed only a small but statistically significant increase in the expression of another gene involved in the same metabolic process, Dgat1, which encodes diacylglycerol $O$-acyltransferase 1 (Supplemental Figure 11). However, we observed no difference in de novo hepatic fatty acid synthesis in vivo in A-CKO mice compared with their littermate controls (Figure 7A). In contrast to L-CKO mice, however, the fatty acid oxidation rate in primary hepatocytes isolated from A-CKO mice was significantly reduced by $31.8 \% \pm$ $10.4 \%$ compared with the rate detected in control mice (Figure $7 \mathrm{~B}$ ). As in L-CKO mice, plasma fatty acid concentrations were also not altered in A-CKO mice (Figure 7C).

To exclude the possibility that the phenotypes of A-CKO mice result from developmental effects, we deleted torsin $\mathrm{A}$ in adult mice by injecting AAV-Cre into the tail veins of 4-month-old mice homozygous for Torla-floxed alleles. We found that torsinA 
levels in liver were reduced by approximately 65\% compared with levels in control mice 4 weeks after virus injection (Figure 8A). Primary hepatocytes isolated from mice injected with AAVCre showed increased lipid droplets in ER-appearing structures (Figure 8B). We also measured newly synthesized and secreted ${ }^{35} \mathrm{~S}$-labeled apoB in primary hepatocytes from AAV-Cre-injected mice (Figure $8 \mathrm{C}$ ). This analysis demonstrated a significant reduction in apoB100 $(59.5 \% \pm 14.7 \%)$ but not apoB48 in the cell lysates; media from these cultures had a significant decrease in the amount of both apoB100 and apoB48 $(91.1 \% \pm 4.7 \%$ and $63.0 \%$ $\pm 6.0 \%$, respectively; Figure $8 \mathrm{D}$ ). These results demonstrate that depletion of torsinA from adult hepatocytes causes hepatocyte lipid accumulation and defects in VLDL synthesis and secretion.

$E R$ stress in livers of $L-C K O$ and A-CKO mice. An association exists between ER stress and defective apoB-mediated TG secretion (26). We therefore examined the ER stress response in livers from $\mathrm{L}-\mathrm{CKO}$ and A-CKO mice. We performed immunoblotting of protein extracts from livers of control and L-CKO mice at 6 months of age to detect glucose-regulated protein 78 (GRP78), $\mathrm{C} / \mathrm{EBP}$ homolog protein (CHOP), phosphorylated eukaryotic initiation factor $2 \alpha$ (p-eIF $2 \alpha$ ), and total eIF $2 \alpha$ (Supplemental Figure 12A). This analysis revealed significant increases in the expression of GRP78, but not CHOP or the ratio of p-eIF $2 \alpha$ to total eIF $2 \alpha$ (Supplemental Figure 12B). We also measured these proteins by immunoblotting liver lysates from A-CKO mice at 6 months of age (Supplemental Figure 12C). GRP78 and p-eIF2 $\alpha$ exhibited increases; CHOP and total eIF2 $\alpha$ were unchanged (Supplemental Figure 12D). IRE1 $\alpha$ activates XBP1 by catalyzing the splicing of its mRNA in response to ER stress (27). We performed an XBP1 splicing assay using cDNAs synthesized from total RNAs isolated from livers of L-CKO mice (Supplemental Figure 12E). We observed no significant differences between L-CKO and control mice (Supplemental Figure 12F). Similar to L-CKO mice, A-CKO mice exhibited no abnormalities in XBP1 splicing of RNA isolated from livers of A-CKO mice (Supplemental Figure 12, G and $\mathrm{H}$ ). Considered together, these experiments indicate that disruption of the torsinA-LAP1 complex in hepatocytes is accompanied by a modest degree of ER stress.

Hepatic insulin resistance in the livers of $L-C K O$ and $A-C K O$ mice. Although glucose tolerance and plasma insulin concentrations were normal in L-CKO and A-CKO mice, indicating the absence of whole-body insulin resistance, we further examined hepatic insulin responsiveness by assessing the activation (phosphorylation) of insulin receptors and AKT2 in livers under physiological conditions. Insulin receptor activation was decreased in the livers of L-CKO mice, but AKT2 activation was not significantly different from that in control mice (Supplemental Figure 13, A and B). In the livers of A-CKO mice, insulin receptor activation was not significantly different than that detected in controls, but AKT2 phosphorylation was significantly decreased (Supplemental Figure 13, $\mathrm{C}$ and $\mathrm{D}$ ). These results suggest that $\mathrm{L}-\mathrm{CKO}$ and $\mathrm{A}-\mathrm{CKO}$ mice may have modest alterations in hepatic insulin responsiveness.

Phosphatidylcholine/phosphatidylethanolamine ratios in livers of $L-C K O$ and A-CKO mice. The hepatic phosphatidylcholine/ phosphatidylethanolamine (PC/PE) ratio influences the dynamics and size limit of lipid droplets, and alterations in it have been linked to impaired VLDL secretion and fatty liver disease (28).
Because of the impaired hepatic VLDL secretion in L-CKO and $\mathrm{A}-\mathrm{CKO}$ mice and the mixed microvesicular and macrovesicular steatosis in A-CKO mice, we examined the livers of both types of mice for altered $\mathrm{PC} / \mathrm{PE}$ ratios. Although the $\mathrm{PC} / \mathrm{PE}$ ratios were not different in $\mathrm{L}-\mathrm{CKO}$ mice compared with ratios in littermate controls, the ratios were significantly decreased in A-CKO mice (Supplemental Table 1). Hence, a decreased PC/PE ratio in the livers of A-CKO mice could have contributed to the impaired VLDL secretion and the microvesicular steatosis we observed.

L-CKO and A-CKO mice on a chow diet develop features of $\mathrm{NASH}$. Chronic hepatic steatosis can cause hepatomegaly. In some instances, chronic steatosis can progress to NASH, which is characterized by steatosis, ballooning degeneration of hepatocytes, and inflammation. Because of the marked steatosis observed in L-CKO and A-CKO mice, we explored whether they developed hepatomegaly and features of NASH. We assessed hepatomegaly by calculating liver-to-body mass ratios. L-CKO mice had a significantly increased liver-to-body mass ratio at 18 months of age (Figure 9A) but no significant difference in the heart-to-body mass ratio (Figure 9B). Serum alanine aminotransferase (ALT) activity, an indicator of hepatocyte damage or death, was nearly 4-fold higher in L-CKO mice than in controls (Figure 9C). Histopathological examination of livers from L-CKO mice revealed steatosis, hepatocyte ballooning, and lobular inflammation, all of which are characteristic histological features of NASH (Supplemental Figure 14A). We observed that hepatic fibrosis was also increased in L-CKO mice compared with controls (Figure 9D). For an objective assessment of NASH features, a liver pathologist blinded to the genotype of the mice assigned NAFLD activity and fibrosis stage scores used in clinical practice (29). Of the $7 \mathrm{~L}-\mathrm{CKO}$ mice examined, 2 had NAFLD activity scores of 5 or higher (consistent with NASH), 4 had a score of 3-4 (borderline NASH), and 1 had a score of 2 (not NASH); all 4 control mice examined had NAFLD activity scores of 2 or lower (not NASH). The mean NAFLD activity score of L-CKO mice was significantly increased compared with that for littermate controls (Figure 9E). L-CKO mice also developed fibrosis by 18 months of age. All 4 control mice had a blindly assigned fibrosis stage of $1 \mathrm{~A}$ (mild, zone 3, perisinusoidal), whereas $1 \mathrm{~L}-\mathrm{CKO}$ mouse had stage 3 (bridging), 3 mice had stage $1 \mathrm{~B}$ (moderate, zone 3 , perisinusoidal), and the remaining 3 mice had stage $1 \mathrm{~A}$ (Supplemental Table 2). Analyses of A-CKO mice demonstrated more severe, accelerated disease. The liver-to-body mass ratio was already significantly increased in 6-month-old A-CKO mice (Figure 9F), with no significant difference in heart-to-body mass ratio (Figure 9G). In A-CKO mice, serum ALT activity was nearly 12-fold higher than that in controls (Figure 9H). Histopathological examination demonstrated hepatic steatosis with a predominance of microvesicular fat, hepatocyte ballooning, and lobular inflammation (Supplemental Figure 14B). By 6 months of age, hepatic fibrosis was already apparent in the livers of A-CKO mice (Figure 9I). Moreover, 6 of 7 A-CKO livers received a NAFLD activity score of 6 or 7 (consistent with NASH) from a pathologist blinded to the genotype, whereas all control livers received NAFLD activity scores of 2 or lower (not NASH) (Figure 9J). Three A-CKO mice were judged to have stage 2 fibrosis, 2 had stage $1 \mathrm{~B}$, and 1 exhibited stage $1 \mathrm{~A}$, as did all controls (Supplemental Table 2). These data demonstrate 
A

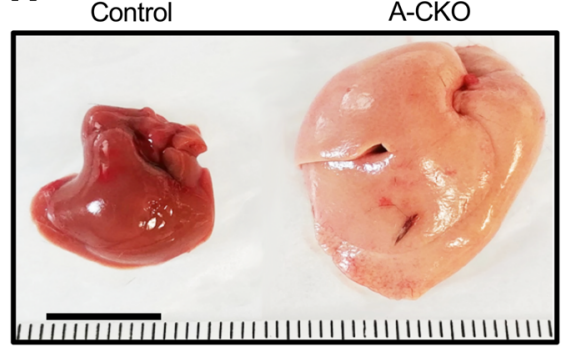

C

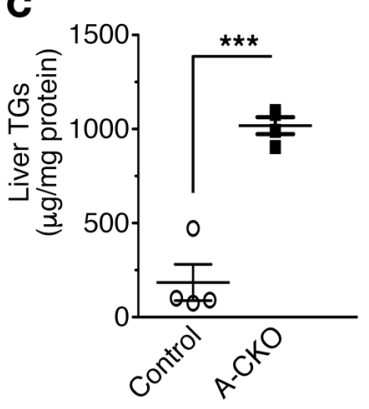

B
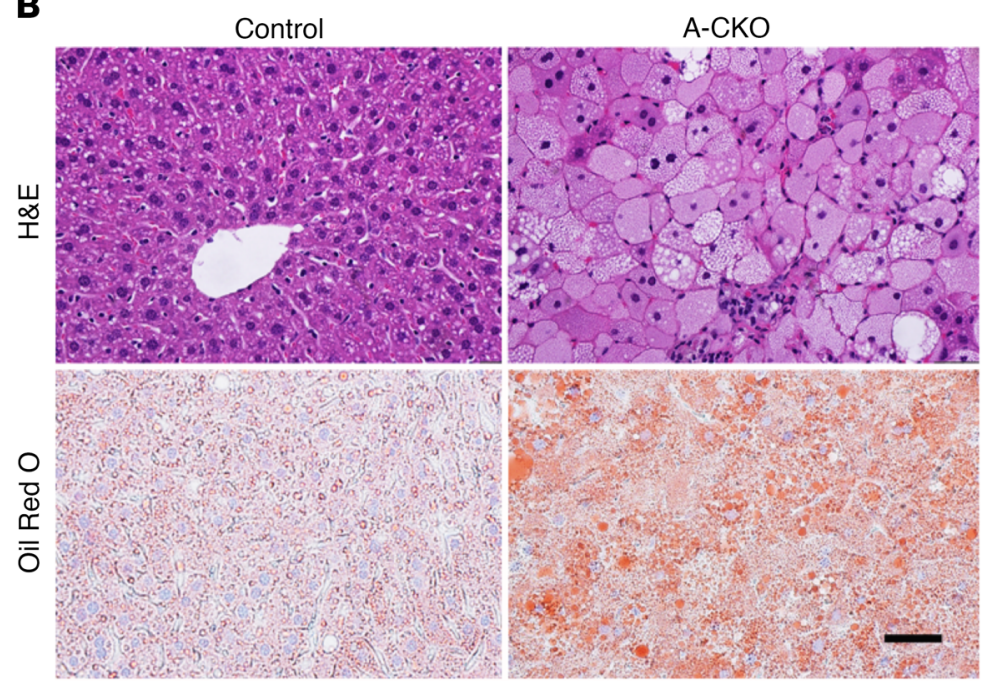

D

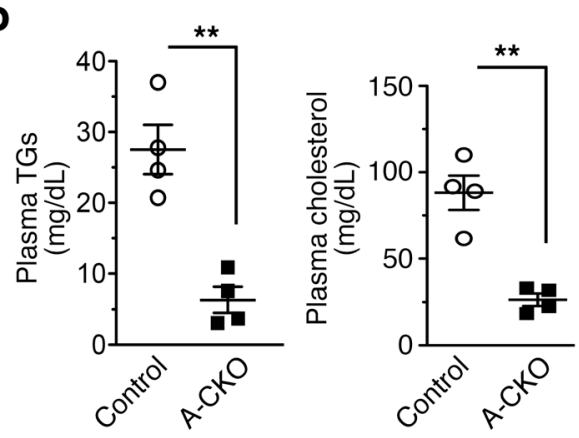

E

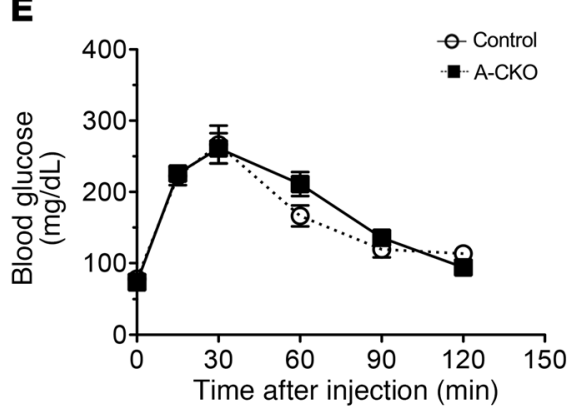

F

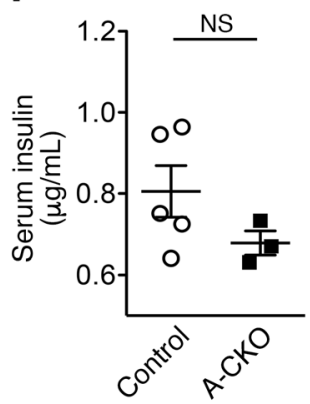

G
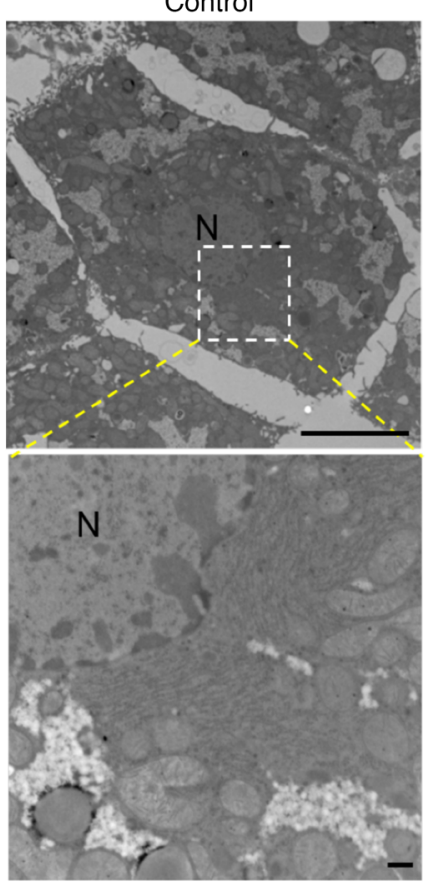

A-CKO

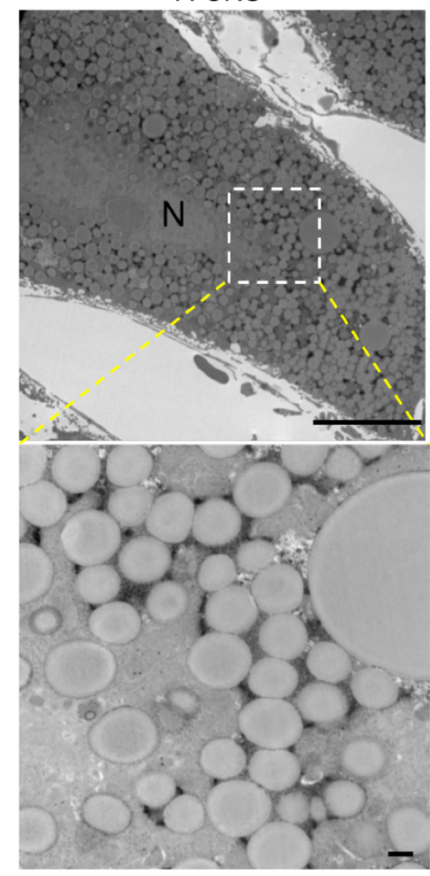

H
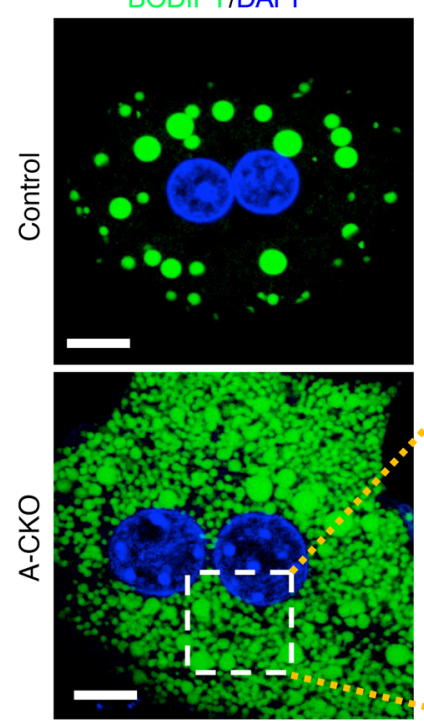

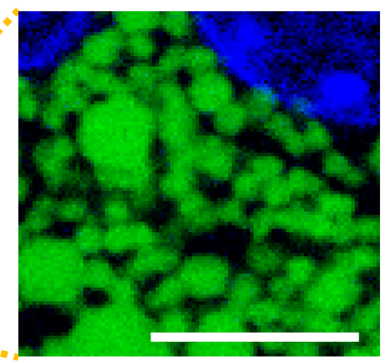


Figure 5. Hepatic steatosis without evidence of insulin resistance in male A-CKO mice fed a chow diet. (A) Photographs of livers from male control (Tor1 $\mathrm{a}^{f /+}$ ) and A-CKO (AlbCre Tor1 $a^{f /-}$ ) mice fed a chow diet. Scale bar: $1 \mathrm{~cm}$. (B) Representative light micrographs of H\&E- and Oil Red O-stained liver sections from chow-fed mice. Scale bar: $50 \mu \mathrm{m}$. (C) Liver TC and cholesterol content. Mice were fasted for 4 to 5 hours before livers were collected ( $n$ $=4$ mice per group). ${ }^{* *} P<0.01$ and ${ }^{* *} P<0.001$, by Student's $t$ test. (D) Plasma TC and cholesterol concentrations ( $n=4$ mice per group). ${ }^{* *} P<$ 0.01 , by Student's $t$ test. (E) Blood glucose concentrations versus time after injection of a glucose bolus into overnight-fasted mice $(n=5$ mice per group). Results were not significantly different at any time point by ANOVA. (F) Serum insulin concentrations. Mice were fasted for 5 hours before plasma was collected ( $n=3-5$ mice per group). $P=$ NS, by Student's $t$ test. (G) Electron micrographs of liver sections from 4-month-old control and A-CKO mice. Top panel shows low-magnification images of a single hepatocyte from control and A-CKO samples. The lower panel is a magnified image of the dashed-line square region in the upper panel. Scale bars: $10 \mu \mathrm{m}$ (upper panel) and $500 \mathrm{~nm}$ (lower panel). N, nucleus. (H) Confocal micrographs of isolated hepatocytes. Lipids were stained with BODIPY (green) and nuclei with DAPI (blue). The right panel is a zoomed image of the dashed-line square region. Scale bars: $10 \mu \mathrm{m}$ (zoom, $10 \mu \mathrm{m})$. In C, D, and $\mathbf{F}$, the values for individual mice are shown, with longer horizontal bars indicating the mean and vertical bars indicating the SEM. Mice used were 4-6 months old.

that both L-CKO and A-CKO mice develop features characteristic of human NASH. Considered together with our other results, these data strongly suggest that LAP1 and torsinA function in a common pathway, the disruption of which disrupts VLDL metabolism, which ultimately can progress to the core histopathological features of human NASH.

\section{Discussion}

NAFLD is highly prevalent and frequently progresses to NASH, representing a major challenge to public health. NAFLD occurs in $25 \%$ to $40 \%$ of the US and European populations, with rates as high as $70 \%$ in individuals with diabetes mellitus (3). Our studies establish a direct role for the torsinA/LAP1 pathway in the regulation of VLDL secretion and liver fat accumulation that progresses to the morphological equivalent of human NASH, including fibrosis. Remarkably, the lipid metabolic derangements resulting from disruption of the torsinA/LAP1 pathway occurred in mice on normal chow diets and in the absence of abnormalities of glucose homeostasis or obesity. These findings highlight nuclear envelope-localized events as key for hepatocyte-autonomous lipid metabolism defects, which are involved in NAFLD development. Consistent with a role in influencing mammalian liver and plasma lipid concentrations, the torsinA/LAP1 pathway was recently identified in an integrative systems genetic analysis of lipid metabolism in inbred mouse strains (30).

The prevalence of NAFLD/NASH is significantly higher in areas where insulin resistance, central obesity, and diabetes are common (31). These comorbidities are often merged under the umbrella of metabolic syndrome (32). There is broad consensus that these features of metabolic syndrome are strongly associated with NAFLD in the majority of affected individuals (33). In contrast, recent work has identified several genetic insults that predispose individuals to NAFLD, NASH, and even cirrhosis in a liver-autonomous manner, unaccompanied by the features of metabolic syndrome $(34,35)$. Our findings identify a nuclear envelope-localized event as a participant in such processes. The identified genes include those encoding proteins involved in lipid droplet biology, such as PNPLA3, encoding patatin-like phospholipase domain-containing protein 3 (36); in de novo lipogenesis, such as GCKR, encoding glucokinase regulatory protein (37); and in the assembly and secretion of VLDL, such as APOB, TM6SF2, encoding transmembrane 6 superfamily member 2, and MTTP, encoding microsomal triglyceride transfer protein (MTP) (3840). All of these genes have been identified as liver-autonomous causes of NAFLD. Although the clinical course of NAFLD in these instances may be affected by the presence of insulin resistance, steatosis may occur even when there are no concomitant systemic abnormalities in carbohydrate or lipid metabolism $(36,41)$.

Another mouse model of significant hepatic steatosis without concomitant insulin resistance was created by antisense oligonucleotide-mediated knockdown of comparative gene identification 58 from hepatocytes. The absence of insulin resistance was attributed to increased compartmentalization of diacylglycerol and PKC $\varepsilon$ in lipid droplets versus the plasma membrane (42). Using the phosphorylation of insulin receptors and AKT2 as indicators of hepatic insulin signaling, we found evidence for modest insulin resistance in both $\mathrm{L}-\mathrm{CKO}$ and $\mathrm{A}-\mathrm{CKO}$ mice. A more detailed investigation of hepatic insulin signaling, including assessment of the subcellular distribution of diacylglycerol and $\mathrm{PKC} \varepsilon$, is warranted.

In addition to its activation by LAP1 in the perinuclear space, torsinA is also activated in the main ER by LULL1 $(17,18)$. The qualitative similarities but clear quantitative differences between mice lacking LAP1 or torsinA in hepatocytes fit with the hypothesis that LAP1 depletion causes only partial torsinA ATPase loss of function because of preserved activation by LULL1 in the main ER. This hypothesis predicts the more severe phenotype we observed with torsinA deletion, which ablated its function from both ER compartments. Our proposed model posits a role for nuclear membrane-localized torsinA ATPase in hepatic lipid homeostasis, particularly for VLDL assembly and secretion. Future studies targeting LULL1 and expressing a constitutively active form of torsinA in hepatocytes of mice with hepatic deletion of LAP1 or LULL1 will help to further test this hypothesis. A-CKO mouse livers also had a decreased PC/PE ratio, whereas the livers of L-CKO mice did not; this could also contribute to the more severely impaired VLDL secretion and greater degree of steatosis, as well as the altered size distribution and abnormal localization of lipid droplets in the A-CKO mice and requires further investigation.

Mice with hepatocyte conditional deletion of LAP1 or torsinA share similarities to mice with loss-of-function of proteins with established functions in VLDL secretion. Homozygous disruption of apoB is embryonically lethal, but heterozygous null mice exhibit decreased plasma cholesterol concentrations $(43,44)$. Mice with hepatocyte-specific depletion of MTP, which transfers TGs into the lumen of the ER for lipidation of apoB, have severely reduced plasma TG, VLDL, and apoB100 concentrations and hepatic steatosis when fed a chow diet, but lack the inflammation characteristic of NASH (45). Livers of these mice have near-complete loss of microsomal apoB100 and no evidence of ER stress (46). Likewise, mice treated with antisense oligonucleotides to inhibit MTP synthesis have reduced hepatic VLDL secretion and steatosis without evidence of ER stress $(47,48)$. In contrast, treatment of adult mice with 
A

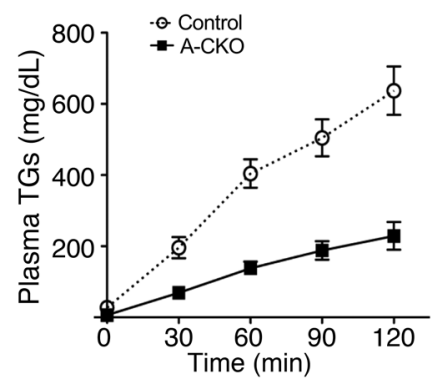

B

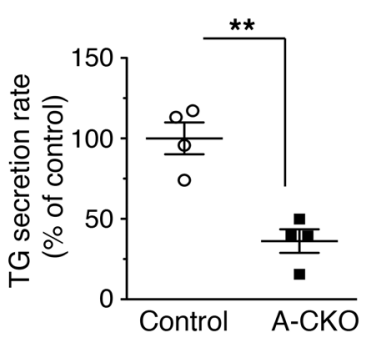

C

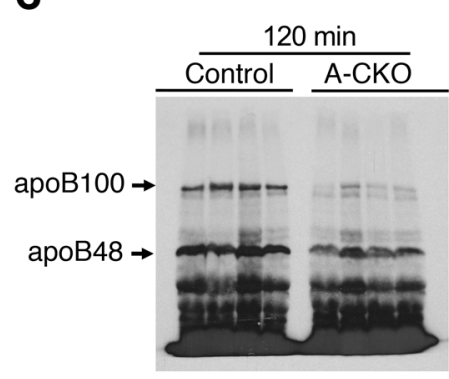

D

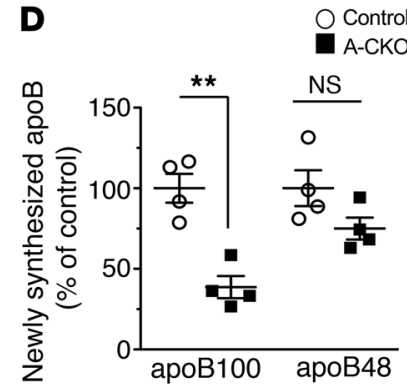

E

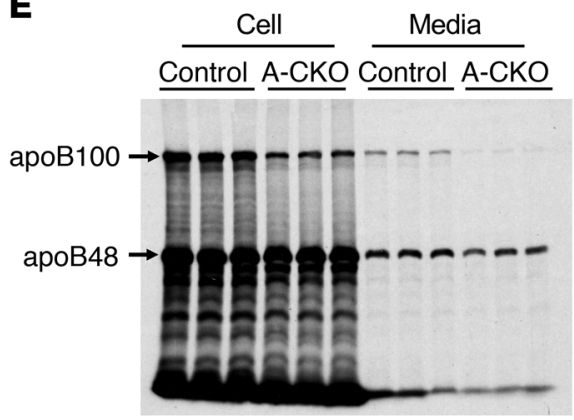

$\mathbf{F}$

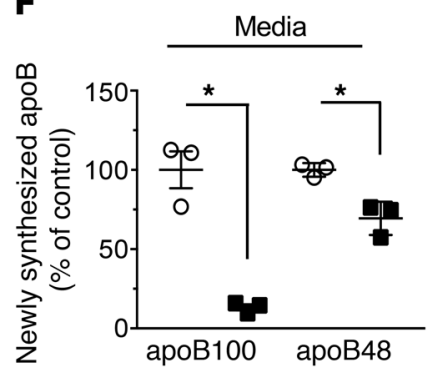

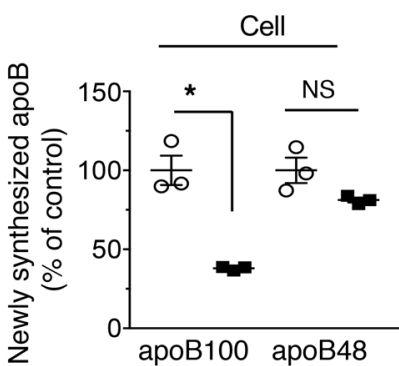

Figure 6. Reduced TC and apoB100 secretion from livers and isolated hepatocytes of A-CKO mice. (A) Mice were injected with tyloxapol to block peripheral TC uptake, and plasma concentrations were measured at the indicated time points. Values represent the mean \pm SEM ( $n=4$ mice per group). (B) TC secretion rates were calculated from the changes in plasma concentrations between 30 and 120 minutes in $\mathbf{A} .{ }^{* *} P<0.01$, by Student's $t$ test. (C) Autoradiogram of SDS-polyacrylamide gel showing ${ }^{35}$ S-labeled plasma proteins collected 120 minutes after i.v. injection with ${ }^{35} \mathrm{~S}$-methionine. Each lane shows plasma proteins from an individual mouse. Migrations of ${ }^{35} \mathrm{~S}$-labeled apoB100 and apoB48 are indicated. (D) Bands corresponding to apoB100 and apoB48 in C were quantified by densitometry, ( $n=4$ mice per group). ${ }^{*} P<0.01$, by Student's $t$ test. (E) Autoradiogram of SDS-polyacrylamide gel showing newly synthesized ${ }^{35} \mathrm{~S}$-labeled proteins in cell lysates and media fractions of primary hepatocyte cultures. Migrations of ${ }^{35} \mathrm{~S}$-labeled apoB100 and apoB48 are indicated ( $n=3$ different hepatocyte cultures from 1 mouse of each genotype). (F) Bands corresponding to apoB100 and apoB48 in $\mathbf{E}$ were quantified by densitometry. The left panel shows results from the media fraction, and the right panel shows results from cell lysate fraction. Results were normalized to the mean values from control samples and set to $100 \%$ ( $n=3$ different hepatocyte cultures from 1 mouse of each genotype). ${ }^{*} P<0.05$, by Student's $t$ test. In B, D, and $\mathbf{F}$, the values for individual mice are shown, with longer horizontal bars indicating the mean and vertical bars indicating the SEM. Mice used were 4 months old.

antisense oligonucleotides against apoB synthesis causes ER stress triggering ER autophagy, followed by lysosomal lipolysis of TGs and fatty acid oxidation that prevent steatosis (48). We observed a modest ER stress response in our mice with hepatic deletion of torsinA or LAP1, with activation in only some of the known pathways. This modest ER stress response probably does not explain the steatosis and defective VLDL secretion seen in these mice.

The effects of the torsinA/LAP1 pathway on VLDL secretion could result from loss of torsinA activity at any of several stages of the assembly and transport of this apoB lipoprotein. ER translocation and lipidation of apoB have several unique features, including the cotranslational ubiquitination and proteasomal degradation that occur in the absence of adequate lipidation of nascent apoB $(49,50)$. Degradation of apoB appears to occur in the livers of mice with hepatic deletion of LAP1 or torsinA, suggesting that torsinA activity may be necessary for its cotranslocational lipidation, functioning along with MTP. However, lipidation of apoB does not appear to require energy (51). TorsinA activity may also be necessary for efficient translocation of apoB across the ER membrane, its proper folding within the lumen of the ER, or its vesicular transport to the Golgi. Loss of torsinA activity would have to be specific for apoB in these processes, as secretion of both albumin and apoA1 was normal in mice with hepatic deletion of either torsinA or LAP1.
Although conditional hepatocyte deletion of LAP1 and conditional hepatocyte deletion of torsinA share essential phenotypes, the 2 situations are not identical. LAP1 hepatocyte deletion causes moderate steatosis, although this is particularly notable, as it occurs on a normal chow diet. These mice exhibit normal rates of hepatic de novo lipogenesis and fatty acid oxidation, as well as normal uptake of fatty acids from the circulation, as indicated by normal plasma fatty acids concentrations. The degree of steatosis therefore appears to be a direct result of the reduction in TG secretion observed in these mice. Unique to this model, however, is the accumulation of some nuclear lipid droplets. Nuclear lipid droplets in hepatocyte cell lines are associated with extension of the inner nuclear membrane into the nucleus (also referred to as the nucleoplasmic reticulum) (52). A potential explanation for nuclear lipid is that LAP1 deletion promotes invaginations of the nuclear envelope, an effect known to occur from alterations in lamins (which bind to LAP1) and other integral inner nuclear membrane proteins $(53,54)$.

There were no nuclear lipid droplets present in torsinA-null hepatocytes, but these mutants exhibited marked abnormalities in the number, size, and location of the cytoplasmic ones, with the vast majority being small and spherical. This phenotype is reminiscent of that of mice with deletion of fat storage-inducing transmembrane protein 2, which functions in the budding of lipid drop- 
A

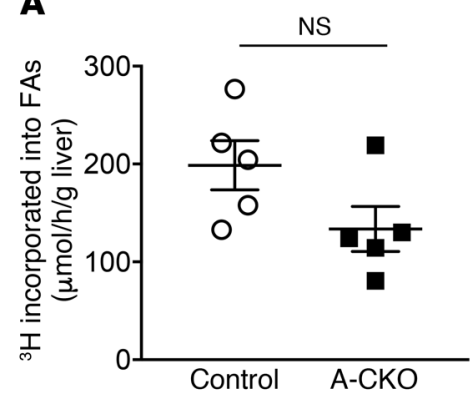

B

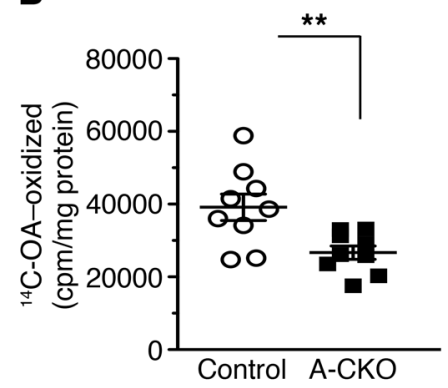

C

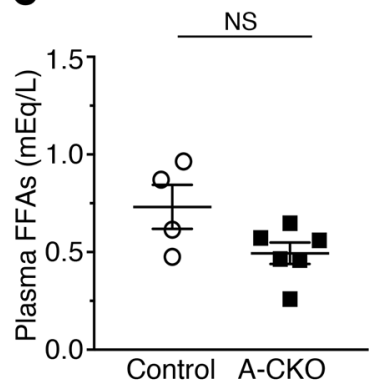

Figure 7. De novo lipogenesis and fatty acid oxidation in livers of A-CKO mice. (A) De novo lipogenesis in livers of control and A-CKO mice. Newly synthesized hepatic lipids were measured by incorporation of ${ }^{3} \mathrm{H}$ into fatty acids 1 hour after i.p. injection with ${ }^{3} \mathrm{H}_{2} \mathrm{O}(n=3$ mice per genotype). $P=\mathrm{NS}$, by Student's $t$ test. (B) Oxidation of fatty acids in hepatocytes isolated from control and A-CKO mice. Oxidative products of ${ }^{14} \mathrm{C}$-oleic acid ${ }^{14} \mathrm{CO}$, and ${ }^{14} \mathrm{C}-\mathrm{labeled}$ acid-soluble metabolites) were measured and normalized to milligrams of cellular protein ( $n=3$ mice per genotype). ${ }^{*} P<0.01$, by Student's $t$ test. (C) Free fatty acids from snap-frozen plasma samples from control and A-CKO mice were measured after a 4- to 5-hour fast. $P=$ NS, by Student's $t$ test. Values for individual mice are shown, with longer horizontal bars indicating the mean and vertical bars indicating the SEM. Mice used were 4-6 months old.

lets from the ER (55). In addition to decreased TG secretion, mice with hepatic deletion of torsinA also had significantly reduced fatty acid oxidation, which likely contributed to the severe steatosis in these mice. Plasma fatty acid concentrations were normal in these mice, suggesting adequate delivery of fatty acids to the liver. Total loss of torsinA activity may therefore lead to defective budding of lipid droplets from the ER and, therefore, decreased delivery of TGs in lipid droplets to mitochondria. This may explain the microvesicular steatosis that was prominent in A-CKO mice.

A role for the torsinA/LAP1 pathway in liver lipid metabolism is not entirely unexpected, given its role in Drosophila melanogaster. Deletion of the torsinA homolog in Drosophila leads to decreased fat body size and increased TG accumulation (56). The fat body shares features with mammalian liver, including the production of an apoB family lipoprotein called lipophorin, the secretion of which requires an MTP homolog (57).

Our studies demonstrate that torsinA and its activator LAP1 profoundly influence VLDL secretion and hepatic steatosis. Deciphering the downstream mechanisms connecting loss of these proteins to the pronounced hepatic phenotypes will be the focus of future research. To our knowledge, the genes encoding these proteins have not been identified in any human GWAS of subjects with NAFLD to date, but rare polymorphisms in these genes with large effects may exist. Direct sequencing of these genes should also be considered in individuals with NAFLD, particularly in the rare individuals lacking insulin resistance. Similarly, human subjects with known mutations in the genes encoding these proteins should be evaluated for hepatic steatosis and serum lipid abnormalities. Finally, our findings point to the torsinA-LAP1 complex as a potential drug target for NAFLD and NASH.

\section{Methods}

Further information can be found in the Supplemental Methods.

Mice. The generation, maintenance, and genotyping of floxed alleles of Lap1 $\left(\operatorname{Lap}^{1 / f f l}\right)$ and Tor1a (Tor1 $\left.1 a^{f / f l}\right)$ mice have been previously described $(16,23)$. Alb-Cre-transgenic mice were purchased from The Jackson Laboratory (stock no. 003574). To generate L-CKO mice, $\mathrm{Alb}-\mathrm{Cre}^{+/-}$mice were crossed with $\mathrm{Lap}^{f / f l l}$ mice to obtain $\mathrm{Alb}-\mathrm{Cre}^{+/-}$ Lap $1^{f /+}$ mice. They were subsequently backcrossed with Lap $1^{f / f l}$ mice to obtain L-CKO $\left(\mathrm{Alb}^{\mathrm{Cl}} \mathrm{Cre}^{+/-} \mathrm{Lap}^{f / f f}\right)$ mice. The genetic background of L-CKO mice was C57BL/6J. The breeding strategy for A-CKO (Alb$\mathrm{Cre}^{+/-} \operatorname{Tor}^{\mathrm{ft}} \mathrm{f}^{\mathrm{fl}-}$ ) and control (Tor1 $\mathrm{a}^{\mathrm{fl}+\mathrm{H}}$ ) mice has been described previously (23). The genetic background of A-CKO mice was a combination of C57BL/6J and 129SvEv. All mice were fertile and produced at the expected Mendelian frequencies. Mice were housed in a climatecontrolled room with a 12-hour light/12-hour dark cycle and fed a regular chow diet (Purina Mills, 5053).

Electron microscopy. Electron microscopy was performed as previously described (16). Briefly, mouse livers were drop-fixed with $1 \%$ paraformaldehyde and $2.5 \%$ glutaraldehyde in $0.1 \mathrm{M}$ cacodylate buffer $(\mathrm{pH}$ 7.4), postfixed with $1 \%$ osmium tetroxide in $0.1 \mathrm{M}$ cacodylate buffer $(\mathrm{pH}$ 7.4), incubated with uranyl acetate, and dehydrated with ethanol. Tissues were subsequently rinsed with propylene oxide and embedded. Sections (60-nm thick) were counterstained with uranyl acetate and lead citrate and examined on a JEM-1200EX electron microscope (JEOL).

Confocal microscopy. Cultured primary hepatocytes on collagencoated coverslips were stained with BODIPY (Thermo Fisher Scientific, D-3922) for 30 minutes. Subsequently, cells were fixed with $4 \%$ paraformaldehyde for 15 minutes at room temperature and then washed and stained with DAPI. Images were obtained using a multiphoton Ti Eclipse confocal microscope (Nikon).

Histology. The mice were euthanized, and the livers were immediately excised and blotted dry. The excised livers were fixed in $10 \%$ formalin for 48 hours. They were then transferred to the Columbia University Histology Core Laboratory for paraffin block preparation and sectioning into $5-\mu \mathrm{m}$ slices. Sections were then stained with H\&E or Picrosirius red (Polysciences, 24901). For Oil Red O staining, livers were transferred onto a $30 \%$ sucrose solution, and frozen sections were prepared at the Columbia University Histology Core. Sections (5- $\mu \mathrm{m}$ thick) were stained with Oil Red O (Polysciences, 25962). Stained sections were photographed using a BX53 upright light microscope attached to a DP72 digital camera (Olympus). To assess NAFLD activity and evaluate fibrosis, a liver pathologist blinded to the mouse genotypes examined sections of the livers stained with H\&E, Oil Red O, and Picrosirius red. The NAFLD activity score has been described previously (29).

Quantification of liver TG and cholesterol. Liver lipids were extracted with a modified Folch method as previously described (58). Briefly, a snap-frozen piece of liver $(100 \mathrm{mg})$ was homogenized in 
A

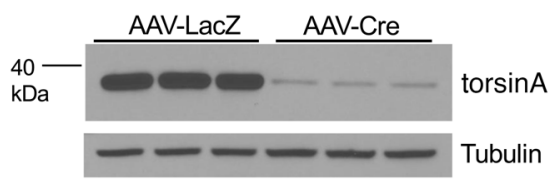

B

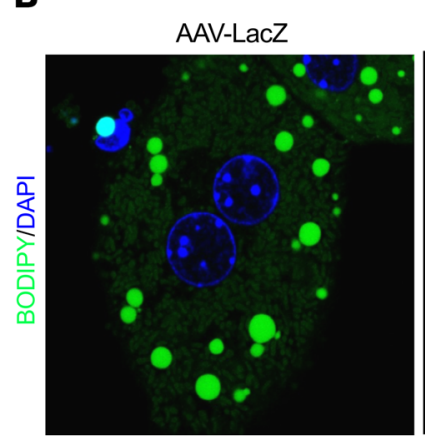

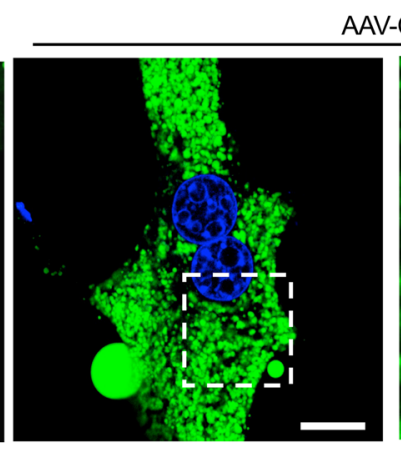

AAV-Cre

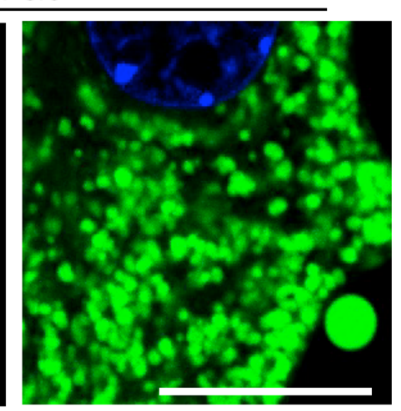

C

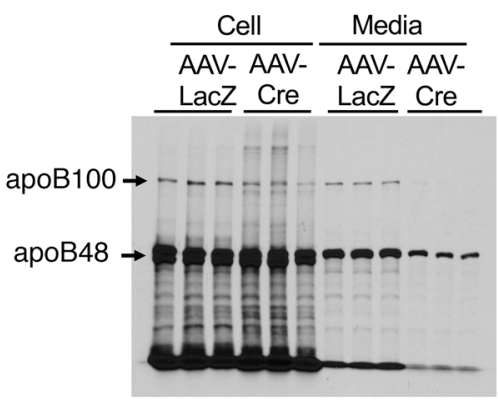

D

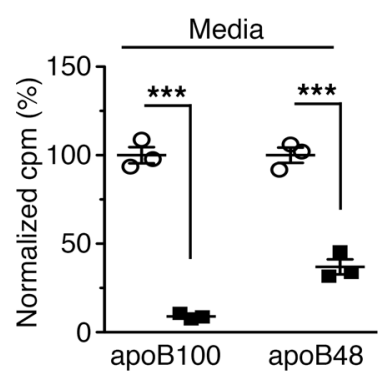

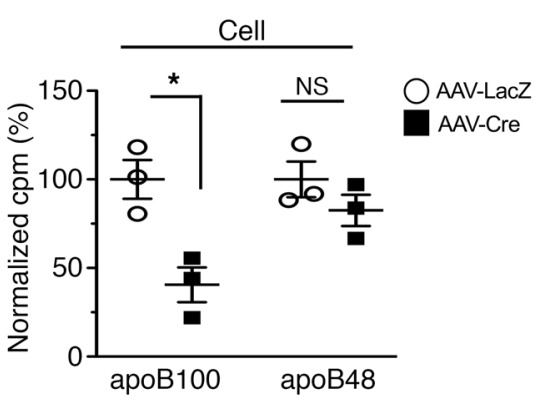

Figure 8. Increased cytoplasmic lipid droplets and reduced apoB100 secretion in hepatocytes isolated from adult mice with "acute" depletion of torsinA. (A) Immunoblot of hepatocyte protein lysates from Tor $7^{f / f l}$ mice injected with AAV-LacZ (control) or AAV-Cre probed with antibodies against torsinA. Hepatocytes were isolated 4 weeks after virus injection. Each lane represents a primary culture of hepatocytes from 1 mouse. Migrations of molecular mass standards are indicated at the left and migrations of torsinA at the right. An immunoblot for $\gamma$-tubulin is shown as a loading control for each sample. (B) Confocal micrographs of isolated hepatocytes from Tor1a ${ }^{f / f l}$ mice injected with AAV-LacZ or AAV-Cre. Lipids were stained with BODIPY (green) and nuclei with DAPI (blue). The far-right panel is a zoomed image of the dashed-line square region. Scale bars: $10 \mu \mathrm{m}$. (C) Autoradiogram showing newly synthesized ${ }^{35} \mathrm{~S}$-labeled proteins in cell lysates and media fractions of primary hepatocytes from Tor1a ${ }^{f / f f}$ mice injected with AAV-LacZ or AAV-Cre. Migrations of ${ }^{35}$ S-methinonine-labeled apoB100 and apoB48 are indicated. (D) Bands corresponding to apoB100 and apoB48 in C were quantified by densitometry. The left panel shows results from the media fraction, and the right panel shows results from cell lysate fraction. Results were normalized to the mean values from AAV-LacZ-injected mice and set to $100 \%$ ( $n=3$ different hepatocyte cultures from 1 mouse of each group). ${ }^{* *} P<0.001$ and ${ }^{*} P<0.05$, by Student's $t$ test. In D, the values for individual mice are shown, with longer horizontal bars indicating the mean and vertical bars indicating the SEM. Mice were 4 months old at the time of virus injection

PBS, and lipids were first extracted with chloroform/methanol (2:1 $\mathrm{v} / \mathrm{v}$ ) and a second time with chloroform/methanol/water (86:14:1 $\mathrm{v} / \mathrm{v} / \mathrm{v})$. The organic layer was dried under nitrogen gas and resolubilized in 15\% Triton X-100 (Sigma-Aldrich) in double-distilled $\mathrm{H}_{2} \mathrm{O}$. TGs and total cholesterol were measured using colorimetric assays (Wako Diagnostics, 461-08992 and 461-09092, respectively).

Blood biochemistries. Plasma TG and cholesterol concentrations were assayed using the colorimetric assays described above. Serum ALT activity and albumin concentration were measured using commercially available kits (Teco Diagnostics A526-120 and SigmaAldrich MAK124, respectively). Serum insulin concentrations were measured using the Mouse Insulin ELISA Kit (Crystal Chem, 90080). For glucose tolerance tests, mice were fasted for 12 hours and given 2 g glucose/kg body weight by i.p. injection. Blood glucose concentrations were measured $0,15,30,60,90$, and 120 minutes after injection using a OneTouch Ultra Glucometer (LifeScan).

Plasma free fatty acid concentrations were measured in snapfrozen plasma samples obtained after a 4 - to 5-hour fast using a commercially available kit (Wako Diagnostics, HR Series NEFA-HR).

Hepatic de novo lipogenesis. Methods to measure de novo lipogenesis have been described previously (59). In brief, mice were injected i.p. with $0.5 \mathrm{mCi}{ }^{3} \mathrm{H}_{2} \mathrm{O}$. After 1 hour, mice were anesthe- tized with ketamine/xylazine, and approximately $250 \mu \mathrm{L}$ blood was collected. Mice were then euthanized and their livers harvested and flash-frozen. Liver tissue $(500 \mathrm{mg}$ ) was incubated with $2.5 \mathrm{M} \mathrm{KOH}$ at $75^{\circ} \mathrm{C}$ for 2 hours. Saponified lipids were isolated by the addition of $80 \%$ ethanol and hexane $(1: 2 \mathrm{v} / \mathrm{v})$. The bottom phase, containing fatty acid salts, was acidified with $3 \mathrm{M} \mathrm{H}_{2} \mathrm{SO}_{4}$. The liberated fatty acids were isolated with additional hexane, dried under nitrogen gas, and resolubilized in $500 \mu \mathrm{L}$ chloroform. Fatty acids were separated by thin-layer chromatography, and the resulting spots were scraped and counted for ${ }^{3} \mathrm{H}$ activity. ${ }^{3} \mathrm{H}$ incorporated into fatty acids was calculated relative to the specific activity of ${ }^{3} \mathrm{H}$ in total body water calculated from the blood sample collected prior to harvesting of the liver.

Fatty acid oxidation. The methods for measuring fatty acid oxidation in mouse primary hepatocytes have been described previously (60). Sixteen hours after plating primary hepatocytes in 6-well plates containing DMEM, the cells were incubated for 2 hours in labeling media (DMEM containing $1.5 \% \mathrm{BSA}, 0.1 \mathrm{mM}$ oleic acid, and $1 \mu \mathrm{Ci} / \mathrm{mL}^{14} \mathrm{C}$-oleic acid). At the end of the labeling and chase periods, media were transferred into 25-mL sealed Erlenmeyer flasks. Lipid oxidation was stopped by adding $200 \mu \mathrm{L} 70 \%$ perchloric acid to the bottom of the flask, thus driving the bicarbonate into $\mathrm{CO}_{2} \cdot{ }^{14} \mathrm{CO}_{2}$ was captured on a piece of $\mathrm{KOH}$ soaked filter paper. After incubating the filter paper in the flask for 1 hour 
A

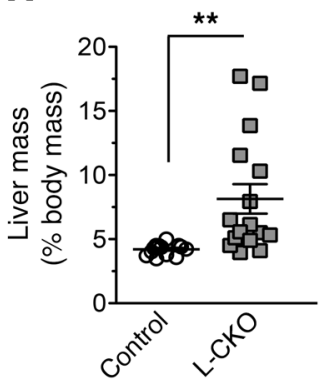

B

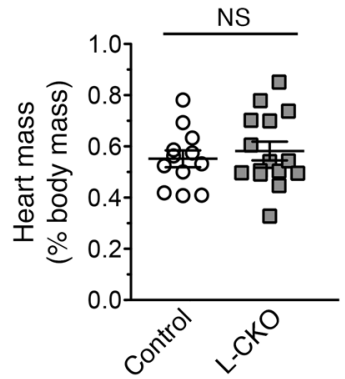

C

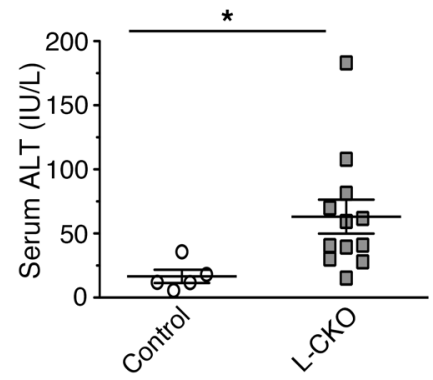

E

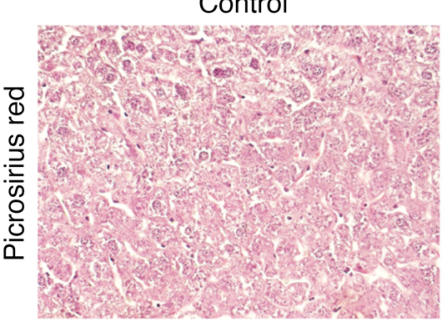

L-CKO

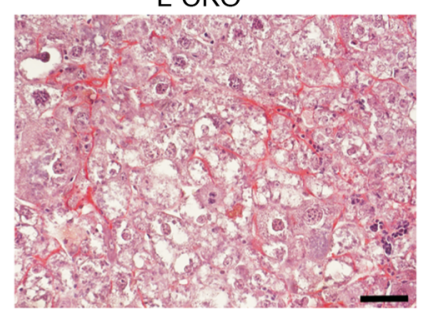

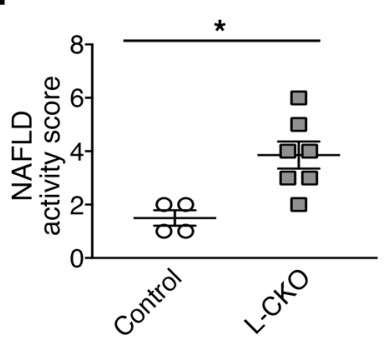

$\mathbf{F}$

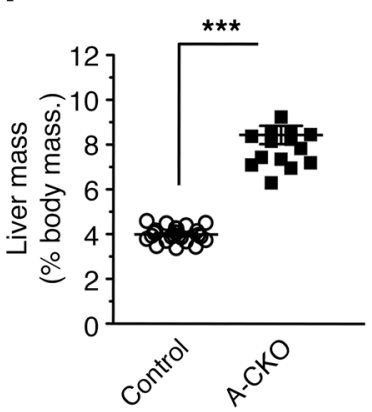

G

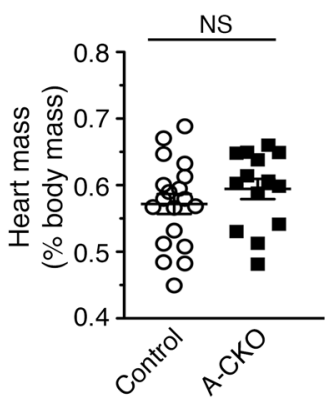

H

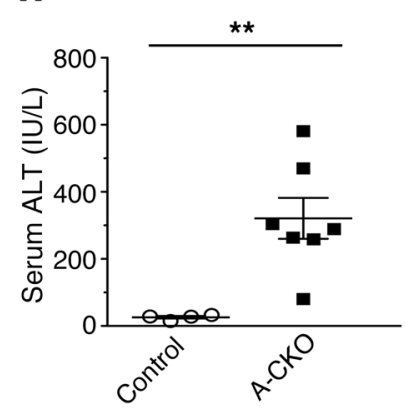

I

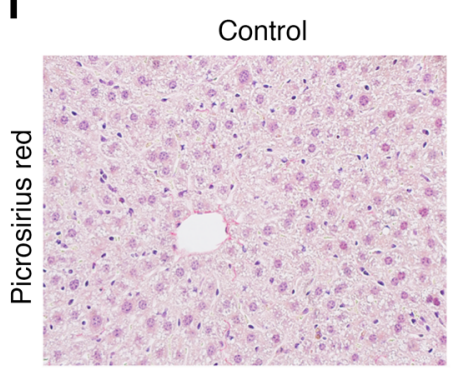

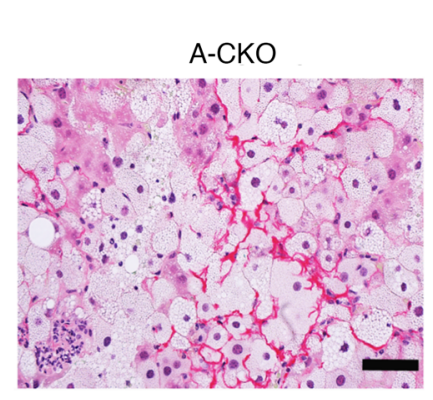

J

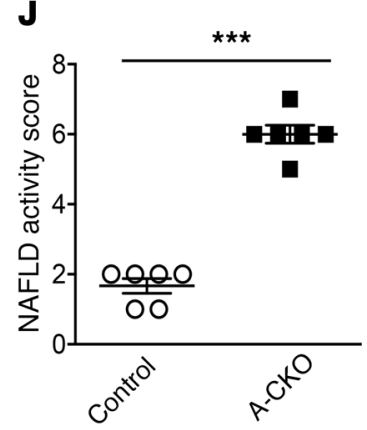

Figure 9. NASH phenotypes in L-CKO and A-CKO mice. (A) Liverto-body mass ratios of control and L-CKO mice ( $n=15$ mice per genotype). ${ }^{* *} P<0.01$, by Student's $t$ test. (B) Heart-to-body mass ratios of control and L-CKO mice ( $n=15$ mice per genotype). $P=$ NS, by Student's $t$ test. (C) Serum ALT activity in control and L-CKO mice ( $n=5-12$ mice per group). ${ }^{*} P<$ 0.05 , by Student's $t$ test. (D) Representative Picrosirius red-stained sections of livers from control and L-CKO mice. Scale bar: $50 \mu \mathrm{m}$. (E) NAFLD activity scores for control and L-CKO mice. ${ }^{*} P<0.05$, by Student's $t$ test. (F) Liver-to-body mass ratios of control and A-CKO mice ( $n=18-20$ mice per genotype). ${ }^{* *} P<0.001$, by Student's $t$ test. (G) Heart-to-body mass ratios of control and A-CKO mice ( $n=15-18$ mice per genotype). $P=$ $N S$, by Student's $t$ test. (H) Serum ALT activity in control and A-CKO mice ( $n=4-7$ mice per group). ${ }^{* *} P$ $<0.01$, by Student's $t$ test. (I) Representative Picrosirius red-stained sections of livers from control and A-CKO mice. Scale bar: $50 \mu \mathrm{m}$. (J) NAFLD activity scores for control and A-CKO mice. ${ }^{* *} P<0.001$, by Student's $t$ test. Except for $\mathbf{D}$ and I, values for individual mice are shown, with longer horizontal bars indicating the mean and vertical bars indicating the SEM. L-CKO mice were 18-24 months old, and A-CKO mice were 6 months old. at room temperature, the filter paper was analyzed by liquid scintillation counting. Media remaining in the flasks were also collected and a fraction counted to measure ${ }^{14} \mathrm{C}$-labeled acid soluble metabolites. After removal of the chase media, cells were scraped off the plates and protein measured using the BCA Protein Assay Kit (Thermo Fisher Scientific). The data were plotted as cpm/mg cellular protein.

In vivo $T G$, apoB, albumin, and apoA1 secretion. In vivo $\mathrm{TG}$ and apoB secretion rates were determined as previously described (48). Briefly, mice were fasted for 4 to 5 hours prior to an i.v. injection of a mixture of $200 \mu \mathrm{Ci}{ }^{35} \mathrm{~S}$-methionine and $500 \mathrm{mg} / \mathrm{kg}$ tyloxapol (SigmaAldrich, T8761-50G) in 0.9\% NaCl. Tyloxapol inhibits both the lipolysis and tissue uptake of lipoproteins in mice, and the accumulation of TGs and ${ }^{35} \mathrm{~S}$-apoB in plasma can be used to estimate the rates of secretion. Blood samples were collected before injection and 30, 60, 90, and 120 minutes after injection of tyloxapol and ${ }^{35} \mathrm{~S}-$ methionine. TG concentrations were measured using the colorimetric assay described above. For apoB secretion rates, whole plasma samples from the 120-minute time point were subjected to $4 \%$ SDSPAGE. The volumes of the samples were adjusted as determined by trichloroacetic acid precipitable ${ }^{35} \mathrm{~S}$-labeled plasma proteins. The gel was dried and exposed to $\mathrm{x}$-ray film to quantitate labeled apoB proteins by densitometry. Hepatic secretion of apoA1 and albumin was measured by subjecting the radiolabeled plasma proteins to $8 \%$ SDSPAGE and performing similar analyses of the dried gels.

Primary hepatocyte isolation. Primary hepatocytes were isolated according to previously described methods (48). Briefly, mice were 
perfused with HBSS without calcium (Thermo Fisher Scientific, 14175-079) and 8 mM HEPES (Thermo Fisher Scientific, 15630-080) via the abdominal vena cava after cutting the portal vein to allow outflow of the perfusate. The hepatocytes were perfused for 8 minutes at a rate of $5 \mathrm{~mL} / \mathrm{min}$ at $37^{\circ} \mathrm{C}$. This was followed by perfusion at the same rate as that for DMEM with $80 \mathrm{mg} / 100 \mathrm{~mL}$ collagenase type I (Worthington Biochemical Corporation CLS-1) for 6 minutes. The liver was removed and minced in a Petri dish containing $4 \mathrm{~mL}$ of the same warm DMEM collagenase mixture for an additional 2 to 4 minutes. Ice-cold DMEM was added and the digested tissue filtered through a nylon mesh and collected in a 50-mL conical tube. The suspension was centrifuged for 5 minutes at $50 \times g$, and the supernatant was aspirated and the cell pellet washed 3 times with $30 \mathrm{~mL}$ ice-cold DMEM. Hepatocytes isolated from A-CKO mice, because of their high-fat content, were handled without using serological pipettes to minimize mechanical stress on the cells. Viable cells were counted after staining with trypan blue, which revealed that more $90 \%$ of cels were viable. The isolated cells were plated onto collagen-coated, 6-well plates at a density of 500,000 cells/well in 4 mL DMEM plus 10\% FBS and cultured for at least 2 hours prior to use for subsequent experiments.

Synthesis and secretion of $а р о B$ in cultured hepatocytes. Isolated primary hepatocytes were plated on collagen-coated, 6-well plates in $10 \%$ FBS and DMEM and $5 \% \mathrm{CO}_{2}$ for 2 hours to allow attachment. Media were changed, and cells were incubated for an additional 12 hours, washed twice with PBS, and cultured for an additional hour in methionine-free DMEM (Thermo Fisher Scientific, 21013-024). Cells were then labeled for 2 hours in methionine-free DMEM containing $150 \mu \mathrm{Ci} / \mathrm{mL}{ }^{35} \mathrm{~S}$-methionine/cysteine (PerkinElmer, NEG072014MC). Media were collected and cells washed twice with ice-cold PBS and then lysed in $1 \mathrm{~mL}$ lysis buffer $(62.5 \mathrm{mM}$ sucrose, $0.5 \%$ sodium deoxycholate, $0.5 \%$ Triton $\mathrm{X}-100,50 \mathrm{mM}$ Tris- $\mathrm{HCl}$, $150 \mathrm{mM} \mathrm{NaCl}, 1 \mathrm{mM}$ benzamidine, $5 \mathrm{mM}$ EDTA, 2 units/mL aprotinin, $50 \mu \mathrm{g} / \mathrm{mL}$ leupeptin, $50 \mu \mathrm{g} / \mathrm{mL}$ pepstatin $\mathrm{A}$, and $10 \mathrm{mM}$ HEPES, $\mathrm{pH}$ 8.0). Immunoprecipitation of apoB100 and apoB48 in cell lysate and media was performed using an anti-apoB antibody (Calbiochem, 178467) followed by separation with $4 \%$ SDS-PAGE. The aliquot of lysate or media used for immunoprecipitation was adjusted to equal quantities of radioactivity as estimated by trichloroacetic acid precipitable ${ }^{35} \mathrm{~S}$-labeled proteins in the lysate. Gels were dried and exposed to $\mathrm{x}$-ray films to quantify apoB100 and apoB48 by densitometry, or bands corresponding to apoB100 and apoB48 were cut from the gel, and radioactivity was quantified by scintillation counting.

$A A V$-mediated hepaticknockdown of LAP1 and torsinA. AAV8 vectors containing a control expression cassette (AAV-TBG-LacZ) or encoding Cre recombinase (AAV-TBG-Cre) were purchased from the University of Pennsylvania Vector Core. For "acute" knockdown experiments, Lap1-floxed (Lap $\left.1^{f / f l}\right)$ and Torla-floxed (Torl $\left.1 a^{f / f l}\right)$ mice were randomly assigned to receive either AAV-TBG-LacZ or AAV-TBG-Cre $\left(1 \times 10^{11}\right.$ genome copies/mouse) via tail vein injection. Protein depletion was confirmed by immunoblotting with specific antibodies.
Statistics. All data are presented as the mean \pm SEM. Statistical methods are described in the figure legends for each data set. An unpaired 2-tailed Student's $t$ test was used to compare differences between any 2 groups, and a 2-way ANOVA was used for multiple comparisons. Statistical significance was set at a $P$ value of less than 0.05 .

Study approval. The IACUCs of Columbia University and the University of Michigan approved all procedures, which were conducted in accordance with the NIH's Guide for the Care and Use of Laboratory Animals (National Academies Press, 2011).

\section{Author contributions}

JYS, AHO, TF, CÖ, SBG, and CCL conducted experiments. MJL performed histopathological analyses. JYS, AHO, TF, WTD, HNG, and HJW contributed to the study's design and analyzed and interpreted data. JYS, WTD, HNG, and HJW wrote the manuscript. WTD, HNG, and HJW supervised the research. All authors reviewed and revised the manuscript.

\section{Acknowledgments}

This work was supported by a New York Nutrition Obesity Research Center Pilot and Feasibility Award (UR002557) as part of a NIH grant (5P3ODK026687, to JYS); NIH training grant T32DK007328 (to SBG); and NIH grants 1R01DK118480 (to WTD, HNG, and HJW), 5R35HL135833 (to HNG), and 5R01AR048997 (to HJW). Image processing and analysis were performed in part in the Confocal and Specialized Microscopy Shared Resource of the Herbert Irving Comprehensive Cancer Center at Columbia University (supported by NIH grant 5P30CA013696), and some of the mouse phenotyping was performed with the help of Anthony S. Ferrante and Jun B. Feranil in the Animal Phenotyping Core of the New York Nutrition Obesity Research Center (supported by NIH grant 5P30DK026687). We thank Kristy Brown (Columbia University) for help with electron microscopy and Renu Nandakumar and Yimeng Xu (Columbia University Biomarker Core Laboratory) for lipidomics analysis.

Address correspondence to: Howard J. Worman or Henry N. Ginsberg, Department of Medicine, Vagelos College of Physicians and Surgeons, Columbia University, 630 West $168^{\text {th }}$ Street, New York, New York 10032, USA. Phone: 212.305.1306; Email: hjw14@ columbia.edu (H.J. Worman). Phone: 212.305.9562; Email: hng1@ cumc.columbia.edu (H.N. Ginsberg). Or to: William T. Dauer, Peter O'Donnell Jr. Brain Institute, University of Texas Southwestern Medical Center, 6000 Harry Hines Boulevard, Room NB5.604, Dallas, Texas 75390-8823, USA. Phone: 214.645.3332; Email: Willliam.dauer@UTSouthwestern.edu.

WTD's present address is: Peter O’Donnell Jr., Brain Institute, University of Texas Southwestern Medical Center, Dallas, Texas, USA.
1. Ginsberg HN, Fisher EA. The ever-expanding role of degradation in the regulation of apolipoprotein B metabolism. JLipid Res. 2009;50 Suppl:S162-S166.

2. Olofsson SO, Borén J. Apolipoprotein B secretory regulation by degradation. Arterioscler Thromb
Vasc Biol. 2012;32(6):1334-1338.

3. Satapathy SK, Sanyal AJ. Epidemiology and natural history of nonalcoholic fatty Liver Disease. Semin Liver Dis. 2015;35(3):221-235.

4. Diehl AM, Day C. Cause, pathogenesis, and treatment of nonalcoholic steatohepatitis. N Engl
JMed. 2017;377(21):2063-2072.

5. Fu S, Watkins SM, Hotamisligil GS. The role of endoplasmic reticulum in hepatic lipid homeostasis and stress signaling. Cell Metab. 2012;15(5):623-634.

6. Dauer WT, Worman HJ. The nuclear envelope 
as a signaling node in development and disease. Dev Cell. 2009;17(5):626-638.

7. Cao H, Hegele RA. Nuclear lamin A/C R482Q mutation in canadian kindreds with Dunnigan-type familial partial lipodystrophy. Hum Mol Genet. 2000;9(1):109-112.

8. Shackleton S, et al. LMNA, encoding lamin A/C, is mutated in partial lipodystrophy. Nat Genet. 2000;24(2):153-156.

9. Speckman RA, et al. Mutational and haplotype analyses of families with familial partial lipodystrophy (Dunnigan variety) reveal recurrent missense mutations in the globular C-terminal domain of lamin A/C. Am J Hum Genet. 2000;66(4):1192-1198.

10. Lüdtke A, et al. Hepatic steatosis in Dunnigan-type familial partial lipodystrophy. Am J Gastroenterol. 2005;100(10):2218-2224.

11. Kwan R, et al. Hepatocyte-specific deletion of mouse lamin A/C leads to male-selective steatohepatitis. Cell Mol Gastroenterol Hepatol. 2017;4(3):365-383

12. Tsai PL, Zhao C, Turner E, Schlieker C. The Lamin B receptor is essential for cholesterol synthesis and perturbed by disease-causing mutations. Elife. 2016;5:e16011.

13. Brady GF, et al. Nuclear lamina genetic variants, including a truncated LAP2, in twins and siblings with nonalcoholic fatty liver disease. Hepatology. 2018;67(5):1710-1725.

14. Kim CE, Perez A, Perkins G, Ellisman MH, Dauer WT. A molecular mechanism underlying the neuralspecific defect in torsinA mutant mice. Proc Natl Acad Sci U S A. 2010;107(21):9861-9866.

15. Senior A, Gerace L. Integral membrane proteins specific to the inner nuclear membrane and associated with the nuclear lamina. JCell Biol. 1988;107(6 Pt 1):2029-2036.

16. Shin JY, et al. Lamina-associated polypeptide-1 interacts with the muscular dystrophy protein emerin and is essential for skeletal muscle maintenance. Dev Cell. 2013;26(6):591-603.

17. Goodchild RE, Dauer WT. The AAA+ protein torsinA interacts with a conserved domain present in LAP1 and a novel ER protein. JCell Biol. 2005;168(6):855-862.

18. Zhao C, Brown RS, Chase AR, Eisele MR, Schlieker C. Regulation of Torsin ATPases by LAP1 and LULL1. Proc Natl Acad Sci U S A. 2013;110(17):E1545-E1554.

19. Kayman-Kurekci G, et al. Mutation in TOR1AIP1 encoding LAP1B in a form of muscular dystrophy: a novel gene related to nuclear envelopathies. Neuromuscul Disord. 2014;24(7):624-633.

20. Ghaoui R, et al. TOR1AIP1 as a cause of cardiac failure and recessive limb-girdle muscular dystrophy. Neuromuscul Disord. 2016;26(8):500-503.

21. Fichtman B, et al. Combined loss of LAP1B and LAP1C results in an early onset multisystemic nuclear envelopathy. Nat Commun. 2019;10(1):605.

22. Ozelius LJ, et al. The early-onset torsion dystonia gene (DYT1) encodes an ATP-binding protein. Nat Genet. 1997;17(1):40-48.

23. Liang CC, Tanabe LM, Jou S, Chi F, Dauer WT. TorsinA hypofunction causes abnormal twisting movements and sensorimotor circuit neurodegeneration. J Clin Invest.
2014;124(7):3080-3092.

24. Davidson NO. Apolipoprotein B mRNA editing: a key controlling element targeting fats to proper tissue. Ann Med. 1993;25(6):539-543.

25. Weisend CM, Kundert JA, Suvorova ES, Prigge JR, Schmidt EE. Cre activity in fetal albCre mouse hepatocytes: Utility for developmental studies. Genesis. 2009;47(12):789-792.

26. Ota T, Gayet C, Ginsberg HN. Inhibition of apolipoprotein B100 secretion by lipid-induced hepatic endoplasmic reticulum stress in rodents. J Clin Invest. 2008;118(1):316-332.

27. Calfon M, et al. IRE1 couples endoplasmic reticulum load to secretory capacity by processing the XBP-1 mRNA. Nature. 2002;415(6867):92-96.

28. van der Veen JN, Kennelly JP, Wan S, Vance JE, Vance DE, Jacobs RL. The critical role of phosphatidylcholine and phosphatidylethanolamine metabolism in health and disease. Biochim Biophys Acta Biomembr. 2017;1859(9 Pt B):1558-1572

29. Kleiner DE, et al. Design and validation of a histological scoring system for nonalcoholic fatty liver disease. Hepatology. 2005;41(6):1313-1321.

30. Parker BL, et al. An integrative systems genetic analysis of mammalian lipid metabolism. Nature. 2019;567(7747):187-193.

31. Younossi ZM, Koenig AB, Abdelatif D, Fazel Y, Henry L, Wymer M. Global epidemiology of nonalcoholic fatty liver disease-Meta-analytic assessment of prevalence, incidence, and outcomes. Hepatology. 2016;64(1):73-84.

32. Grundy SM. Metabolic syndrome update. Trends Cardiovasc Med. 2016;26(4):364-373.

33. Jinjuvadia R, Antaki F, Lohia P, Liangpunsakul $\mathrm{S}$. The association between nonalcoholic fatty liver disease and metabolic abnormalities in the United States population. J Clin Gastroenterol. 2017;51(2):160-166.

34. Dongiovanni P, Romeo S, Valenti L. Genetic factors in the pathogenesis of nonalcoholic fatty liver and Steatohepatitis. Biomed Res Int 2015;2015:460190.

35. Di Filippo M, et al. Homozygous MTTP and APOB mutations may lead to hepatic steatosis and fibrosis despite metabolic differences in congenital hypocholesterolemia. J Hepatol. 2014;61(4):891-902.

36. Romeo S, et al. Genetic variation in PNPLA3 confers susceptibility to nonalcoholic fatty liver disease. Nat Genet. 2008;40(12):1461-1465.

37. Sliz E, et al. NAFLD risk alleles in PNPLA3, TM6SF2, GCKR and LYPLAL1 show divergent metabolic effects. Hum Mol Genet. 2018;27(12):2214-2223.

38. Kozlitina J et al. Exome-wide association study identifies a TM6SF2 variant that confers susceptibility to nonalcoholic fatty liver disease. Nat Genet. 2014;46(4):352-356.

39. Schonfeld G, Lin X, Yue P. Familial hypobetalipoproteinemia: genetics and metabolism. Cell Mol Life Sci. 2005;62(12):1372-1378.

40. Wetterau JR, et al. Absence of microsomal triglyceride transfer protein in individuals with abetalipoproteinemia. Science. 1992;258(5084):999-1001.

41. Stender S, Kozlitina J, Nordestgaard BG, Tybjærg-Hansen A, Hobbs HH, Cohen JC. Adiposity amplifies the genetic risk of fatty liver disease conferred by multiple loci. Nat Genet.
2017;49(6):842-847.

42. Cantley JL, et al. CGI-58 knockdown sequesters diacylglycerols in lipid droplets/ER-preventing diacylglycerol-mediated hepatic insulin resistance. Proc Natl Acad Sci U S A . 2013;110(5):1869-1874.

43. Farese RV, Ruland SL, Flynn LM, Stokowski RP, Young SG. Knockout of the mouse apolipoprotein $B$ gene results in embryonic lethality in homozygotes and protection against diet-induced hypercholesterolemia in heterozygotes. Proc Natl Acad Sci U S A. 1995;92(5):1774-1778.

44. Huang LS, Voyiaziakis E, Markenson DF, Sokol KA, Hayek T, Breslow JL. apo B gene knockout in mice results in embryonic lethality in homozygotes and neural tube defects, male infertility, and reduced HDL cholesterol ester and apo A-I transport rates in heterozygotes. J Clin Invest. 1995;96(5):2152-2161.

45. Raabe M, et al. Analysis of the role of microsomal triglyceride transfer protein in the liver of tissue-specific knockout mice. J Clin Invest. 1999;103(9):1287-1298.

46. Liao W, Hui TY, Young SG, Davis RA. Blocking microsomal triglyceride transfer protein interferes with apoB secretion without causing retention or stress in the ER. J Lipid Res. 2003;44(5):978-985.

47. Lee RG, et al. Comparison of the pharmacolog ical profiles of murine antisense oligonucleotides targeting apolipoprotein B and microsomal triglyceride transfer protein. J Lipid Res. 2013;54(3):602-614.

48. Conlon DM, et al. Inhibition of apolipoprotein B synthesis stimulates endoplasmic reticulum autophagy that prevents steatosis. JClin Invest. 2016;126(10):3852-3867.

49. Zhou M, Fisher EA, Ginsberg HN. Regulated co-translational ubiquitination of apolipoprotein B100. A new paradigm for proteasomal degradation of a secretory protein. J Biol Chem. 1998;273(38):24649-24653.

50. Fisher EA, et al. The degradation of apolipoprotein $\mathrm{B} 100$ is mediated by the ubiquitin-proteasome pathway and involves heat shock protein 70. J Biol Chem. 1997;272(33):20427-20434.

51. Sirwi A, Hussain MM. Lipid transfer proteins in the assembly of apoB-containing lipoproteins. JLipid Res. 2018;59(7):1094-1102.

52. Ohsaki Y, Kawai T, Yoshikawa Y, Cheng J, Jokitalo E, Fujimoto T. PML isoform II plays a critical role in nuclear lipid droplet formation. J Cell Biol. 2016;212(1):29-38

53. Ellenberg J, et al. Nuclear membrane dynamics and reassembly in living cells: targeting of an inner nuclear membrane protein in interphase and mitosis. J Cell Biol. 1997;138(6):1193-1206.

54. Goulbourne CN, Malhas AN, Vaux DJ. The induction of a nucleoplasmic reticulum by prelamin A accumulation requires CTP:phosphocholine cytidylyltransferase- $\alpha$. J Cell Sci. 2011;124(Pt 24):4253-4266.

55. Choudhary V, Ojha N, Golden A, Prinz WA. A conserved family of proteins facilitates nascent lipid droplet budding from the ER. J Cell Biol. 2015;211(2):261-271.

56. Grillet M, et al. Torsins are essential regulators of cellular lipid metabolism. Dev Cell. 2016;38(3):235-247. 
57. Palm W, et al. Lipoproteins in Drosophila melanogaster--assembly, function, and influence on tissue lipid composition. PLoS Genet. 2012;8(7):e1002828.

58. Folch J, LeesM, Sloane Stanley GH. A simple method for the isolation and purification of total lipides from animal tissues. J Biol Chem. 1957;226(1):497-509.

59. Zhang YL, et al. Aberrant hepatic expression of PPARgamma2 stimulates hepatic lipogenesis in a mouse model of obesity, insulin resistance, dyslipidemia, and hepatic steatosis. J Biol Chem.
2006;281(49):37603-37615.

60. Erol E, Kumar LS, Cline GW, Shulman GI, Kelly DP, Binas B. Liver fatty acid binding protein is required for high rates of hepatic fatty acid oxidation but not for the action of PPARalpha in fasting mice. FASEB J. 2004;18(2):347-349. 